\title{
Balanced split sets and Hamilton-Jacobi equations
}

\author{
Pablo Angulo Ardoy \\ Department of Mathematics \\ Universidad Autónoma de Madrid. \\ Luis Guijarro \\ Department of Mathematics \\ Universidad Autónoma de Madrid. \\ ICMAT CSIC-UAM-UCM-UC3M
}

November 20, 2018

\begin{abstract}
We study the singular set of solutions to Hamilton-Jacobi equations with a Hamiltonian independent of $u$. In a previous paper, we proved that the singular set is what we called a balanced split locus. In this paper, we find and classify all balanced split loci, identifying the cases where the only balanced split locus is the singular locus, and the cases where this does not hold. This clarifies the relationship between viscosity solutions and the classical approach of characteristics, providing equations for the singular set. Along the way, we prove more structure results about the singular sets.
\end{abstract}

\section{Introduction}

In this paper we consider the following boundary value problem:

$$
\begin{array}{rl}
H(p, d u(p))=1 & p \in \Omega \\
u(p)=g(p) & p \in \partial \Omega
\end{array}
$$

for a smooth compact manifold $\Omega$ of dimension $n$ with boundary, $H$ smooth, $H^{-1}(1) \cap T_{p}^{*} \Omega$ strictly convex for every $p$, and $g$ smooth and satisfying the compatibility condition:

$$
|g(y)-g(z)|<d(y, z) \quad \forall y, z \in \partial \Omega
$$

where $d$ is the distance induced by the Finsler metric:

$$
\varphi_{p}(v)=\sup \left\{\langle v, \alpha\rangle_{p}: \alpha \in T_{p}^{*} \Omega, H(p, \alpha)=1\right\}
$$

This definition gives a norm in every tangent space $T_{p} \Omega$. Indeed, $H$ is a norm at every tangent space if we make the harmless assumption that $H$ is positively homogeneous of order $1: H(p, \lambda \alpha)=$ $\lambda H(p, \alpha)$ for $\lambda>0$.

A unique viscosity solution is given by the Lax-Oleinik formula:

$$
u(p)=\inf _{q \in \partial \Omega}\{d(p, q)+g(q)\}
$$

A local classical solution can be computed near $\partial \Omega$ following characteristic curves, which are geodesics of the metric $\varphi$ starting from a point in $\partial \Omega$ with initial speed given by a vector field on 
$\partial \Omega$ that is determined by $H$ and $g$ (see 3.1): if $\gamma:[0, t) \rightarrow \Omega$ is the unique (projected) characteristic from a point $q \in \partial \Omega$ to $p=\gamma(t)$ that does not intersect Sing, then $u(p)=g(q)+t$. The viscosity solution can be thought of as a way to extend the classical solution to the whole $\Omega$.

Let Sing be the closure of the singular set of the viscosity solution $u$ to the above problem. Sing has a key property: any point in $\Omega \backslash$ Sing can be joined to $\partial \Omega$ by a unique characteristic curve that does not intersect Sing. A set with this property is said to split $\Omega$. Once characteristic curves are known, if we replace Sing by any set $S$ that splits $\Omega$, we can still apply the formula in the last paragraph to obtain another function with some resemblance to the viscosity solution (see definition 2.4).

The set Sing has an extra property: it is a balanced split locus. This notion, introduced in [AG] and inspired originally by the paper [IT], is related to the notion of semiconcave functions that is now common in the study of Hamilton-Jacobi equations (see section 2.1). Our goal in this paper is to determine whether there is a unique balanced split locus. In the cases when this is not true, we also give an interpretation of the multiple balanced split loci.

Finally, we recall that the distance function to the boundary in Riemannian and Finsler geometry is the viscosity solution of a Hamilton-Jacobi equation ([MM]), and the cut locus is the closure of the singular set of the distance function to the boundary ([LN]). Thus, our results also apply to cut loci in Finsler geometry.

\subsection{Outline}

In section 2 we state our results, give examples, and comment on possible extensions. Section 3 gathers some of the results from the literature we will need, and includes a few new lemmas that we use later. Section 4 contains our proof that the distance to a balanced split locus and distance to the $k$-th conjugate point are Lipschitz. Section 5 contains the proof of the main theorems, modulo a result that is proved in section 6 . This last section also features detailed descriptions of a balanced split set at each of the points in the classification introduced in $\mathrm{AG}$.

\subsection{Acknowledgements.}

The authors express their gratitude to Ireneo Peral, Yanyan Li, Luc Nguyen, Marco Fontelos and Juan Carlos Álvarez for interesting conversations about this problem. The paper also benefited greatly from the referee's input, and the authors want to thank him for it. Both authors were partially supported during the preparation of this work by grants MTM2007-61982 and MTM200802686 of the MEC and the MCINN respectively.

\section{Statement of results.}

\section{$2.1 \quad$ Setting}

We study a Hamilton-Jacobi equation given by (1.1) and (1.2) in a $C^{\infty}$ compact manifold with boundary $\Omega$. $H$ is smooth and strictly convex in the second argument and the boundary data $g$ is smooth and satisfies (1.3).

The solution by characteristics gives the characteristic vector field on $\partial \Omega$, which we write as a map $\Gamma: \partial \Omega \rightarrow T \Omega$ that is a section of the projection map $\pi: T \Omega \rightarrow \Omega$ of the tangent to $\Omega$. The characteristic curves are the integral curves of the geodesic vector field in $T \Omega$ with initial point 
$\Gamma(z)$ for $z \in \partial \Omega$. The projected characteristics are the projection to $\Omega$ of the characteristics. The characteristic vector field is smooth and points inwards (see 3.1).

Let $\Phi$ be the geodesic flow in $T \Omega$, and $D(\Phi)$ its domain. We introduce the set $V \subset \mathbb{R} \times \partial \Omega$ :

$$
V=\{x=(t, z), z \in \partial \Omega, t \geq 0,(t, \Gamma(z)) \in D(\Phi)\}
$$

$V$ has coordinates given by $z \in \partial \Omega$ and $t \in \mathbb{R}$. We set $F: V \rightarrow \Omega$ to be the map given by following the projected characteristic with initial value $\Gamma(z)$ a time $t$ :

$$
F(t, z)=\pi(\Phi(t, \Gamma(z)))
$$

The vector $r$ given as $\frac{\partial}{\partial t}$ in the above coordinates maps under $F$ to the tangent to the projected characteristic.

Definition 2.1. For a set $S \subset \Omega$, let $A(S) \subset V$ be the set of all $x=(t, z) \in V$ such that $F(s, z) \notin S, \forall 0 \leq s<t$. We say that a set $S \subset \Omega$ splits $\Omega$ iff $F$ restricts to a bijection between $A(S)$ and $\Omega \backslash S$.

Whenever $S$ splits $\Omega$, we can define a vector field $R_{p}$ in $\Omega \backslash S$ to be $d F_{x}\left(r_{x}\right)$ for the unique $x$ in $A(S)$ such that $F(x)=p$.

Definition 2.2. For a point $a \in S$, we define the limit set $R_{a}$ as the set of vectors in $T_{a} \Omega$ that are limits of sequences of the vectors $R_{p}$ defined above at points $p \in \Omega \backslash S$.

Definition 2.3. If $S$ splits $\Omega$, we also define a set $Q_{p} \subset V$ for $p \in \Omega$ by

$$
Q_{p}=\left(F \mid \frac{}{A(S)}\right)^{-1}(p)
$$

The following relation holds between the sets $R_{p}$ and $Q_{p}$ :

$$
R_{p}=\left\{d F_{x}\left(r_{x}\right): x \in Q_{p}\right\}
$$

Definition 2.4. If $S$ splits $\Omega$, we can define a real-valued function $h$ in $\Omega \backslash S$ by setting:

$$
h(p)=g(z)+t
$$

where $(t, z)$ is the unique point in $A(S)$ with $F(t, z)=p$.

If we start with the viscosity solution $u$ to the Hamilton-Jacobi equations, and let $S=$ Sing be the closure of the set where $u$ is not $C^{1}$, then $S$ splits $\Omega$. If we follow the above definition involving $A(S)$ to get a new function $h$, then we find $h=u$.

Definition 2.5. A set $S$ that splits $\Omega$ is a split locus iff

$$
S=\overline{\left\{p \in S: \quad \sharp R_{p} \geq 2\right\}}
$$

The role of this condition is to restrict $S$ to its essential part. A set that merely splits $\Omega$ could be too big: actually $\Omega$ itself splits $\Omega$. The following lemma may clarify this condition.

Lemma 2.6. A set $S$ that splits $\Omega$ is a split locus if and only if $S$ is closed and it has no proper closed subsets that split $\Omega$. 
Proof. The only if part is trivial, so we will only prove the other implication. Assume $S$ is a split locus and let $S^{\prime} \subset S$ be a closed set splitting $\Omega$. Let $q \in S \backslash S^{\prime}$ a point with $\sharp R_{q} \geq 2$. Since $S^{\prime}$ is closed, there is a neighborhood of $q$ away from $S^{\prime}$; so, if $\gamma_{1}$ is a segment of a geodesic in $\Omega \backslash S^{\prime}$ joining $\partial \Omega$ with $q$, there is a point $q_{1}$ in $\gamma_{1}$ lying beyond $q$. Furthermore, we can choose the point $q_{1}$ not lying in $S$, so there is a second geodesic $\gamma_{2}$ contained in $\Omega \backslash S \subset \Omega \backslash S^{\prime}$ from $\partial \Omega$ to $q_{1}$. As $q \in S$, we see $\gamma_{2}$ is necessarily different from $\gamma_{1}$, which is a contradiction if $S^{\prime}$ is split. Therefore we learn $S^{\prime} \supset\left\{p \in S: \quad \sharp R_{p} \geq 2\right\}$, so $S=\overline{\left\{p \in S: \quad \sharp R_{p} \geq 2\right\}} \subset S^{\prime}$.

Finally, we introduce the following more restrictive condition (see 3.3 for the definition of $v_{p}(q)$, the vector from $p$ to $q$, and 3.2 for the Finsler dual of a vector).

Definition 2.7. We say a split locus $S \subset \Omega$ is balanced for given $\Omega, H$ and $g$ (or simply that it is balanced if there is no risk of confusion) iff for all $p \in S$, all sequences $p_{i} \rightarrow p$ with $v_{p_{i}}(p) \rightarrow$ $v \in T_{p} \Omega$, and any sequence of vectors $X_{i} \in R_{p_{i}} \rightarrow X_{\infty} \in R_{p}$, then

$$
w_{\infty}(v)=\max \left\{w(v), w \text { is dual to some } R \in R_{p}\right\}
$$

where $w_{\infty}$ is the dual of $X_{\infty}$.

Remark. We proved in $\mathrm{AG}$ that the cut locus of a submanifold in a Finsler manifold and the closure of the singular locus of the solution to (1.1) and (1.2) are always balanced split loci. The proof (and the definition of balanced itself) was inspired by the paper [IT], and consists basically of an application of the first variation formula.

We give now another proof that relates the balanced condition to the notion of semiconcave functions, which is now common in the study of Hamilton-Jacobi equations. More precisely, we simply translate theorem 3.3.15 in the book CS to our language to get the following lemma:

Lemma 2.8. The closure of the singular set of the viscosity solution to (1.1) and (1.2) is a balanced split locus.

Proof. Let $u$ be the viscosity solution to (1.1) and (1.2), and let Sing be the closure of its singular set. We leave to the reader the proof that Sing is a split locus (otherwise, see [AG]).

It is well known that $u$ is semiconcave (see for example [CS, 5.3.7]). The superdifferential $D^{+} u(p)$ of $u$ at $p$ is the convex hull of the set of limits of differentials of $u$ at points where $u$ is $C^{1}$ (see [CS, 3.3.6]). At a point where $u$ is $C^{1}$, the dual of the speed vector of a characteristic is the differential of $u$. Thus, the superdifferential at $p$ is the convex hull of the duals to the vectors in $R_{p}$. We deduce:

$$
\max \left\{w(v), w \text { is dual to some } R \in R_{p}\right\}=\max \left\{w(v), w \in D^{+} u(p)\right\}
$$

Given $p \in \Omega$, and $v \in T_{p} \Omega$, the exposed face of $D^{+} u(p)$ in the direction $v$ is given by:

$$
D^{+}(p, v)=\left\{\tilde{w} \in D^{+} u(p): \tilde{w}(v) \leq w(v) \forall w \in D^{+} u(p)\right\}
$$

The balanced condition can be rephrased in these terms as:

Let $p_{i} \rightarrow p \in S$ be a sequence with $v_{p_{i}}(p) \rightarrow v \in T_{p} \Omega$, and let $w_{i} \in D^{+} u\left(p_{i}\right)$ be a sequence converging to $w \in D^{+} u(p)$.

Then $w \in D^{+} u(p,-v)$ 
which is exactly the statement of theorem [CS, 3.3.15], with two minor remarks:

1. The condition is restricted to points $p \in S$. At points in $\Omega \backslash S$, the balanced condition is trivial.

2. In the balanced condition, we use the vectors $v_{p_{i}}(p)$ from $p_{i}$ to $p$, contrary to the reference [CS]. Thus the minus sign in the statement.

In the light of this new proof, we can regard the balanced condition as a differential version of the semiconcavity condition. A semiconcave function that is a solution to (1.1) is also a viscosity solution (see [CS, 5.3.1]). This papers tries to recover the same result under the balanced condition.

\subsection{Results}

For fixed $\Omega, H$ and $g$ satisfying the conditions stated earlier, there is always at least one balanced split locus, namely the singular set of the solution of (1.1) and (1.2). In general, there might be more than one balanced split loci, depending on the topology of $\Omega$.

Our first theorem covers a situation where there is uniqueness.

Theorem 2.9. Assume $\Omega$ is simply connected and $\partial \Omega$ is connected.

Then there is a unique balanced split locus, which is the singular locus of the solution of (1.1) and (1.2).

The next theorem removes the assumption that $\partial \Omega$ is connected, at the price of losing uniqueness:

Theorem 2.10. Assume $\Omega$ is simply connected and $\partial \Omega$ has several connected components. Let $S \subset \Omega$ be a balanced split locus.

Then $S$ is the singular locus of the solution of (1.1) and (1.2) with boundary data $g+a$ where the function $a$ is constant at each connected component of $\partial \Omega$.

The above theorem describes precisely all the balanced split loci in a situation where there is non-uniqueness. If $\Omega$ is not simply connected, the balanced split loci are more complicated to describe. We provide a somewhat involved procedure using the universal cover of the manifold. However, the final answer is very natural in light of the examples.

Theorem 2.11. There exists a bijection between balanced split loci for given $\Omega, H$ and $g$ and an open subset of the homology space $H^{1}(\Omega, \partial \Omega)$ containing zero.

In fact, this theorem follows immediately from the next, where we construct such bijection:

Theorem 2.12. Let $\widetilde{\Omega}$ be the universal cover of $\Omega$, and lift both $H$ and $g$ to $\widetilde{\Omega}$.

Let $a:[\partial \widetilde{\Omega}] \rightarrow \mathbb{R}$ be an assignment of a constant to each connected component of $\partial \widetilde{\Omega}$ that is equivariant for the action of the automorphism group of the covering and such that $\widetilde{g}(z)+a(z)$ satisfies the compatibility condition (1.3) in $\widetilde{\Omega}$. Then the singular locus $\widetilde{S}$ of the solution $\widetilde{u}$ to:

$$
\begin{gathered}
\widetilde{H}(x, d \widetilde{u}(x))=1 \quad x \in \widetilde{\Omega} \\
\widetilde{u}(x)=\widetilde{g}(x)+a(z) \quad x \in \partial \widetilde{\Omega}
\end{gathered}
$$

is invariant by the automorphism group of the covering, and its quotient is a set $S$ that is a balanced split locus for $\Omega, H$ and $g$. Furthermore: 
1. The procedure above yields a bijection between balanced split loci for given $\Omega, H$ and $g$ and equivariant compatible functions $a:[\partial \widetilde{\Omega}] \rightarrow \mathbb{R}$.

2. Among the set of equivariant functions $a:[\partial \widetilde{\Omega}] \rightarrow \mathbb{R}$ (that can be identified naturally with $H^{1}(\Omega, \partial \Omega)$ ), those compatible correspond to an open subset of $H^{1}(\Omega, \partial \Omega)$ that contains 0 .

Remark. The space $H^{1}(\Omega, \partial \Omega)$ is dual to $H_{n-1}(\Omega)$ by Lefschetz theorem. The proof of the above theorems rely on the construction from $S$ of a $(n-1)$-dimensional current $T_{S}$ that is shown to be closed and thus represents a cohomology class in $H_{n-1}(\Omega)$. The proof of the above theorem also shows that the map sending $S$ to the homology class of $T_{S}$ is a bijection from the set of balanced split loci onto a subset of $H_{n-1}(\Omega)$.

In order to prove the above we will make heavy use of some structure results for balanced split loci. To begin with, we use the results of [AG], which were stated for a balanced split locus with this paper in mind. In the last section, we improve the description of the cut locus near each of these types of points.

We also study some very important functions for the study of the cut locus. Recall the global coordinates in $V$ given by $z \in \partial \Omega$ and $t \in \mathbb{R}$. Let $\lambda_{j}(z)$ be the value of $t$ at which the geodesic $s \rightarrow \Phi(s, z)$ has its $j$-th conjugate point (counting multiplicities), or $\infty$ if there is no such point. Let $\rho_{S}: \partial \Omega \rightarrow \mathbb{R}$ be the minimum $t$ such that $F(t, z) \in S$.

Lemma 2.13. All functions $\lambda_{j}: \partial \Omega \rightarrow \mathbb{R}$ are Lipschitz continuous.

Lemma 2.14. The function $\rho_{S}: \partial \Omega \rightarrow \mathbb{R}$ is Lipschitz continuous if $S$ is balanced.

Both results were proven in [IT for Riemannian manifolds, and the second one was given in [LN]. Thus, our results are not new for a cut locus, but the proof is different from the previous ones and may be of interest. We have recently known of another proof that $\rho$ and $\lambda_{1}$ are Lipschitz ([CR]).

\section{$2.3 \quad$ Examples}

Take as $\Omega$ any ring in a euclidean $n$-space bounded by two concentric spheres. Solve the HamiltonJacobi equations with $H(x, p)=|p|$ and $g=0$. The solution is the distance to the spheres, and the cut locus is the sphere concentric to the other two and equidistant from each of them. However, any sphere concentric to the other two and lying between them is a balanced split set, so there is a one parameter family of split balanced sets. When $n>2$, this situation is a typical application of 2.10. In the $n=2$ case, there is also only one free parameter, which is in accord with 2.12, as the rank of the $H_{1}$ homology space of the ring is one.

For a more interesting example, we study balanced split sets with respect to a point in a euclidean torus. We take as a model the unit square in the euclidean plane with its borders identified. It is equivalent to study the distance with respect to a point in this euclidean torus, or the solution to Hamilton-Jacobi equations with respect to a small distance sphere centered at the point with the Hamiltonian $H(p)=|p|$ and $g=0$.

A branch of cleave points (see 3.5) must keep constant the difference of the distances from either sides (recall prop 7.2 in $\mathrm{AG}$, or read the beginning of section [5). Moving to the covering plane of the torus, we see they must be segments of hyperbolas. A balanced split locus is the union of the cleave segments and a few triple or quadruple points. The set of all balanced split loci is a 2-parameter family, as predicted by our theorem 2.12 . 


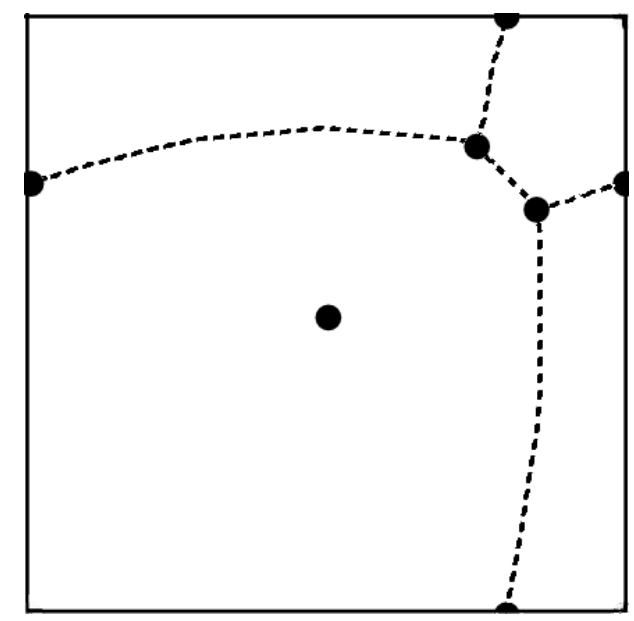

Figure 1: Balanced split set in a torus

\subsection{Extensions}

The techniques in this paper could be applied to other first order PDEs, or systems of PDEs. In particular, we can mention the Cauchy problem with a Hamiltonian dependent on $t$, and both Cauchy and Dirichlet problems with a Hamiltonian dependent on $u$. Characteristic curves are well behaved on those cases (though some extra hypothesis are needed for a Hamiltonian dependent on $u$ ).

In particular, we believe our proofs of 2.13 and 2.14 are more easily extensible to other settings than the previous ones in the literature. This may simplify the task of proving that the singular locus for other PDEs have locally finite $n-1$ Hausdorff measure.

In this paper and its predecessor $[\mathrm{AG}$, strong regularity assumptions were assumed. There are powerful reasons to weaken the regularity assumptions when studying Hamilton-Jacobi equations. The definition of a balanced split locus itself does not require strong regularity. Less regular data, though, could produce qualitatively different behavior. In the structure result 3.5, the dimensions of the sets of points of each type may become higher, as a consequence of the general Morse-SardFederer theorem (see $[\mathrm{F}]$ ). Also, if $g$ is not $C^{1}$, we can expect non-trivial intersections between the singular set and $\partial \Omega$, or rarefaction waves.

\section{Preliminaries}

\subsection{Definitions}

Definition 3.1. Let $v \in T_{p} \Omega$ be a tangent vector at $p$ in a Finsler manifold $(\Omega, \varphi)$. The Riemannian metric at $(p, v)$ is given by:

$$
g_{(p, v)}(X, Y)=\frac{\partial^{2} \varphi}{\partial v^{i} v^{j}}(p, v) X^{i} Y^{j}
$$

Definition 3.2. The dual one form to a vector $X \in T_{p} \Omega$ with respect to a Finsler metric $\varphi$ is the unique one form $w \in T_{p}^{*} \Omega$ such that $w(X)=\varphi(X)^{2}$ and $\left.w\right|_{H}=0$, where $H$ is the hyperplane tangent to the level set

$$
\left\{Y \in T_{p} \Omega, \varphi(Y)=\varphi(X)\right\}
$$


at $X$. It coincides with the usual definition of dual one form in Riemannian geometry.

For a vector field, the dual differential one-form is obtained by applying the above construction at every point.

We will often use the notation $\widehat{X}$ for the dual one-form to the vector $X$.

In coordinates, the dual one form $w$ to the vector $X$ is given by:

$$
w_{j}=\frac{\partial \varphi}{\partial v^{j}}(p, X)
$$

and also, in terms of the Riemannian metric at $(p, X)$ :

$$
w(\cdot)=g_{(p, X)}(X, \cdot)
$$

With this notion of dual form, we can restate the usual equations for the characteristic vector field at points $p \in \partial \Omega$ :

$$
\begin{gathered}
\varphi_{p}\left(X_{p}\right)=1 \\
\left.\widehat{X_{p}}\right|_{T(\partial \Omega)}=d g \\
X_{p} \text { points inwards }
\end{gathered}
$$

Definition 3.3. Whenever there is a unique unit speed minimizing geodesic $\gamma$ joining the points $p$ and $q$ in $\Omega$, we define, following [IT],

$$
v_{p}(q)=\dot{\gamma}(0)
$$

For fixed $p \in \Omega$, then any $q$ sufficiently close to $p$ is joined to $p$ by a unique unit speed minimizing geodesic, so $v_{p}(q)$ is well defined.

Definition 3.4. Let $z \in \partial \Omega$ and $x=(t, z) \in V$.

We say $x$ is conjugate iff $F$ is not a local diffeomorphism at $x$. The order of conjugacy is the dimension of the kernel of $d F$.

We say $x$ is a first conjugate vector iff no point $(s, x)$ for $s<t$ is conjugate.

We recall from [AG] a result on the structure of balanced split loci (in that paper, conjugate points are called focal points):

Theorem 3.5. A balanced split locus consists of the following types of points:

- Cleave points: Points at which $R_{p}$ consists of two non-conjugate vectors. The set of cleave points is a smooth hypersurface;

- Edge points: Points at which $R_{p}$ consists of exactly one conjugate vector of order 1 . This is a set of Hausdorff dimension at most $n-2$;

- Degenerate cleave points: Points at which $R_{p}$ consists of two vectors, such that one of them is conjugate of order 1, and the other may be non-conjugate or conjugate of order 1. This is a set of Hausdorff dimension at most $n-2$;

- Crossing points: Points at which $R_{p}$ consists of non-conjugate and conjugate vectors of order 1 , and $R_{p}^{*}$ spans an affine subspace of dimension $2\left(R_{p}^{*}\right.$ is the set of duals to vectors in $\left.R_{p}\right)$. This is a rectifiable set of dimension $n-2$;

- Remainder: A set of Hausdorff dimension at most $n-3$; 


\subsection{Special coordinates}

In $[\mathrm{AG}$, we used only a few properties of the exponential map essentially introduced in [W]. Those properties, stated in proposition 8.3 of [AG], were shown enough to guarantee the existence of special coordinates for $F$ near a conjugate point of order $k$ (see the paragraph on special coordinates before theorem 6.3 of $\left[\mathrm{AG}\right.$ ]). Near a point $x^{0} \in V$ and its image $F\left(x^{0}\right) \in \Omega$, we can find coordinates such that $x^{0}$ has coordinates 0 , and $F$ is written as

$$
F\left(x_{1}, \ldots, x_{n}\right)=\left(x_{1}, \ldots, x_{n-k}, F_{n-k+1}(x), \ldots, F_{n}(x)\right)
$$

where

- $\frac{\partial}{\partial x_{i}} F_{j}\left(x^{0}\right)$ is 0 for any $i$ and $n-k+1 \leq j \leq n$,

- $\frac{\partial}{\partial x_{i}} \frac{\partial}{\partial x_{1}} F_{j}\left(x^{0}\right)$ is $\delta_{j}^{i}$, for $n-k+1 \leq i \leq n$ and $n-k+1 \leq j \leq n$.

- $\frac{\partial}{\partial x_{1}}\left(x^{0}\right)=r_{x^{0}}$

\subsection{Lagrangian submanifolds of $T^{*} \Omega$}

Let $D$ be the homeomorphism between $T \Omega$ and $T^{*} \Omega$ induced by the Finsler metric as in definition 3.2 ( $D$ is actually a $C^{\infty}$ diffeomorphism away from the zero section). We define a map:

$$
\Delta(t, z)=D(\Phi(t, \Gamma(z)))
$$

and a subset of $T^{*} \Omega$ :

$$
\Theta=\Delta(V)
$$

where $\Phi$ is the geodesic flow in $T \Omega$. This is a smooth $n$-submanifold of $T^{*} \Omega$ with boundary.

It is a standard fact that, for a function $u: \Omega \rightarrow \mathbb{R}$, the graph of its differential $d u$ is a Lagrangian submanifold of $T^{*} \Omega$. The subset of $\Theta$ corresponding to small $t$ is the graph of the differential of the solution $u$ to the HJ equations by characteristics. Indeed, all of $\Theta$ is a lagrangian submanifold of $T^{*} \Omega$ (see [D]).

We can also carry over the geodesic vector field from $T \Omega$ into $T^{*} \Omega$ (outside the zero sections). This vector field in $T^{*} \Omega$ is tangent to $\Theta$. Then, as we follow an integral curve $\gamma(t)$ within $\Theta$, the tangent space to $\Theta$ describes a curve $\lambda(t)$ in the bundle $G$ of lagrangian subspaces of $T^{*} \Omega$. It is a standard fact that the vector subspace $\lambda(t) \subset T_{\gamma(t)}^{*} \Omega$ intersects the vertical subspace of $T_{\gamma(t)}^{*} \Omega$ in a non-trivial subspace for a discrete set of times. We will review this fact, in elementary terms, and prove a lemma that will be important for the proof of lemma 2.14.

Let $\eta(t)$ be an integral curve of $r$ with $x_{0}=\eta(0)$ a conjugate point of order $k$. In special coordinates near $x_{0}$, for $t$ close to 0 , the differential of $F$ along $\eta$ has the form:

$$
d F(\eta(t))=\left(\begin{array}{cc}
I_{n-k} & 0 \\
* & *
\end{array}\right)=\left(\begin{array}{cc}
I_{n-k} & 0 \\
0 & 0
\end{array}\right)+t\left(\begin{array}{cc}
0 & 0 \\
0 & I_{k}
\end{array}\right)+\left(\begin{array}{cc}
0 & 0 \\
* & R(t)
\end{array}\right)+\left(\begin{array}{cc}
0 & 0 \\
* & E(t)
\end{array}\right)
$$

where $\left|R^{\prime}(t)\right|<\varepsilon$ and $|E|<\varepsilon$, with $E=0$ if $\gamma(0)=x_{0}$.

Let $w \in \operatorname{ker} d F\left(\eta\left(t_{1}\right)\right)$ and $v \in \operatorname{ker} d F\left(\eta\left(t_{2}\right)\right)$ be unit vectors in the kernel of $d F$ for $t_{1}<t_{2}$ close to 0 . It follows that both $v$ and $w$ are spanned by the last $k$ coordinates. We then find:

$$
0=w \cdot d F\left(\eta\left(t_{2}\right)\right) \cdot v-v \cdot d F\left(\eta\left(t_{1}\right)\right) \cdot w=\left(t_{2}-t_{1}\right) w \cdot v+w\left(R\left(t_{2}\right)-R\left(t_{1}\right)\right) v+w\left(E\left(t_{2}\right)-E\left(t_{1}\right)\right) v
$$


and it follows (for some $t_{1}<t^{*}<t_{2}$ ):

$$
\left(t_{2}-t_{1}\right) w \cdot v<|w||v|\left(\left|R^{\prime}\left(t^{*}\right)\right|+2 \varepsilon\right)\left(t_{2}-t_{1}\right)<3 \varepsilon\left(t_{2}-t_{1}\right)
$$

or

$$
w \cdot v<3 \varepsilon
$$

This also shows that the set of $t$ 's such that $d F(\eta(t))$ is singular is discrete.

Say the point $x_{0}=\left(z_{0}, t_{0}\right)$ is the $j$-th conjugate point along the integral curve of $r$ through $x_{0}$ from $z_{0}$, and recall that it is of order $k$ as conjugate point. As $z$ moves towards $z_{0}$, all functions $\lambda_{j}(z), \ldots, \lambda_{j+k}(z)$ converge to $t_{0}$. Let $z_{i}$ be a sequence of points converging to $z_{0}$ such that the integral curve through $z_{i}$ meets its $k$ conjugate points near $z_{0}$ at $M$ linear subspaces (e.g. $\lambda_{j}\left(z_{i}\right)=$ $\left.\cdots=\lambda_{j+k_{1}}\left(z_{i}\right) ; \lambda_{j+k_{1}+1}\left(z_{i}\right)=\cdots=\lambda_{j+k_{2}}\left(z_{i}\right) ; \ldots ; \lambda_{j+k_{M-1}+1}\left(z_{i}\right)=\cdots=\lambda_{j+k_{M}}\left(z_{i}\right)\right)$. we get the following theorem (see also lemma 1.1 in [IT]):

Lemma 3.6. The subspaces ker $d_{\left(\lambda_{j+k_{l}}\left(z_{i}\right), z_{i}\right)} F$ for $l=1, \ldots, M$ converge to orthogonal subspaces of $\operatorname{ker} d_{\left(\lambda_{j}\left(z_{0}\right), z_{0}\right)} F$, for the standard inner product in the special coordinates at the point $\left(\lambda_{j}\left(z_{0}\right), z_{0}\right)$.

\subsection{A useful lemma}

Lemma 3.7. Let $U$ be an open set in $\mathbb{R}^{n}, A \subset U$ a proper open set, $C^{+}$an open cone, $V \subset U$ an arbitrary open set and $\varepsilon>0$ such that at any point $q \in \partial A \cap V$, we have $\left(q+C^{+}\right) \cap\left(q+B_{\varepsilon}\right) \subset A$.

Then $\partial A \cap V$ is a Lipschitz hypersurface. Moreover, for any vector $X \in C^{+}$, take coordinates so that $X=\frac{\partial}{\partial x_{1}}$. Then $\partial A \cap V$ is a graph $S=\left\{\left(h\left(x_{2}, . ., x_{n}\right), x_{2}, . ., x_{n}\right)\right\}$ for a Lipschitz function $h$.

Proof. Choose the vector $X \in C^{+}$and coordinate system in the statement. Assume $X$ has norm 1 , so that $q+t X \in q+B_{t}$ for small positive $t$. Take any point $p \in \partial A \cap V$. We notice that all points $p+t \frac{\partial}{\partial x_{1}}$ for $0<t<\varepsilon$ belong to $A$, and all points $p+t \frac{\partial}{\partial x_{1}}$ for $-\varepsilon<t<0$ belongs to $U \backslash A$. Indeed, there cannot be a point $p+t \frac{\partial}{\partial x_{1}} \in A$ for $-\varepsilon<t<0$ because the set $\left(p+t \frac{\partial}{\partial x_{1}}\right)+\left(C^{+} \cap B_{\varepsilon}\right)$ contains an open neighborhood of $p$, which contains points not in $A$. In particular, there is at most one point of $\partial A \cap V$ in each line with direction vector $\frac{\partial}{\partial x_{1}}$.

Take two points $q_{1}, q_{2} \in \mathbb{R}^{n-1}$ sufficiently close and consider the lines $L_{1}=\left\{\left(t, q_{1}\right), t \in \mathbb{R}\right\}$ and $L_{2}=\left\{\left(t, q_{2}\right), t \in \mathbb{R}\right\}$. Assume there is a $t_{1}$ such that $\left(t_{1}, q_{1}\right)$ belongs to $\partial A$. If there is no point of $\partial A$ in $L_{2}$ then either all points of $L_{2}$ belong to $A$ or they belong to $U \backslash A$. Both of these options lead to a contradiction if $\left(\left(t_{1}, q_{1}\right)+C^{+}\right) \cap\left(\left(t_{1}, q_{1}\right)+B_{\varepsilon}\right) \cap L_{2} \neq \emptyset$ (this condition is equivalent to $K\left|q_{1}-q_{2}\right|<\varepsilon$ for a constant $K$ that depends on $C^{+}$and the choice of $X \in C^{+}$and the coordinate system).

Thus there is a point $\left(t_{2}, q_{2}\right) \in \partial A$. For the constant $K$ above and $t \geq t_{1}+K\left|q_{1}-q_{2}\right|$, the point $\left(t, q_{2}\right)$ lies in the set $\left(t_{1}, q_{1}\right)+C^{+}$, so we have

$$
t_{2}<t_{1}+K\left|q_{1}-q_{2}\right|
$$

The points $q_{1}$ and $q_{2}$ are arbitrary, and the lemma follows.

\subsection{Some generalities on HJ equations.}

Lemma 3.8. For fixed $\Omega$ and $H$, two functions $g, g^{\prime}: \partial \Omega \rightarrow \mathbb{R}$ have the same characteristic vector field in $\partial \Omega$ iff $g^{\prime}$ can be obtained from $g$ by addition of a constant at each connected component of $\partial \Omega$. 
Proof. It follows from (3.1) that $g$ and $g^{\prime}$ have the same characteristic vector field at all points if and only $d g=d g^{\prime}$ at all points.

For our next definition, observe that given $\Omega, H$ and $g$, we can define a map $\tilde{u}: V \rightarrow \mathbb{R}$ by $\tilde{u}(t, z)=t+g(z)$.

Definition 3.9. We say that a function $u: \Omega \rightarrow \mathbb{R}$ is made from characteristics iff $\left.u\right|_{\partial \Omega}=g$ and $u$ can be written as $u(p)=\tilde{u} \circ s$ for a (not necessarily discontinuous) section $s$ of $F: V \rightarrow \Omega$.

Remark. In the paper $\mathrm{Me}$, the same idea is expressed in different terms: all characteristics are used to build a multi-valued solution, and then some criterion is used to select a one-valued solution. The criterion used there is to select the characteristic with the minimum value of $\tilde{u}$.

Lemma 3.10. The viscosity solution to (1.1) and (1.2) is the unique continuous function that is made from characteristics.

Proof. Let $h$ be a function made from characteristics, and $u$ be the function given by formula (1.5). Let Sing be the closure of the singular set of $u$.

Take a point $z \in \partial \Omega$. Define:

$$
t_{z}^{*}=\sup \{t \geq 0: h(F(\tau, z))=u(F(\tau, z)) \forall 0 \leq \tau<t\}
$$

Claim: $t_{z}^{*}<\rho_{\text {Sing }}(z)$ implies $h$ is discontinuous at $F\left(t_{z}^{*}, z\right)$.

Proof of the claim: Assume that $t_{z}^{*}<\rho_{\text {Sing }}(z)$ and $h$ is continuous at $F\left(t_{z}^{*}, z\right)$ for some $z \in \partial \Omega$.

As $t_{z}^{*}<\rho_{\text {Sing }}(z)<\lambda_{1}(z)$, there is an open neighborhood $O$ of $\left(t_{z}^{*}, z\right)$ such that $\left.F\right|_{O}$ is a diffeomorphism onto a neighborhood of $p=F\left(t_{z}^{*}, z\right)$.

By hypothesis, there is a sequence $t_{n} \rightarrow t_{z}^{*}$ and $p_{n}=F\left(t_{n}, z\right)$ such that $h\left(p_{n}\right) \neq u\left(p_{n}\right)$. As $h$ is built from characteristics using a section $s$, we have $h\left(p_{n}\right)=\tilde{u}\left(s\left(p_{n}\right)\right)=\tilde{u}\left(\left(s_{n}, y_{n}\right)\right)=s_{n}+g\left(y_{n}\right)$, for $\left(s_{n}, y_{n}\right) \neq\left(t_{n}, z\right)$.

For $n$ big enough, the point $\left(s_{n}, y_{n}\right)$ does not belong to $O$, as $\left(t_{n}, z\right)$ is the only preimage of $p_{n}$ in $O$. As $h\left(p_{n}\right) \rightarrow h(p)$, and $\partial \Omega$ is compact, we deduce the $s_{n}$ are bounded. We can take a subsequence of $\left(s_{n}, y_{n}\right)$ converging to $\left(s_{\infty}, y_{\infty}\right) \notin O$. So we have $p=F\left(t_{z}^{*}, z\right)=F\left(s_{\infty}, y_{\infty}\right)$. If $p \notin \operatorname{Sing}$, we deduce that $\lim _{n \rightarrow \infty} h\left(p_{n}\right)=\tilde{u}\left(s_{\infty}, y_{\infty}\right)>h(p)=u(p)=\tilde{u}\left(t_{z}^{*}, z\right)$, so $h$ is discontinuous at $p$.

Using the claim, we conclude the proof: if $h$ is continuous, then $\rho_{\text {Sing }}(z) \leq t_{z}^{*}$ for all $z \in \partial \Omega$, and $u=h$, as any point in $\Omega$ can be expressed as $F(t, z)$ for some $z$, and some $t \leq \rho_{\text {Sing }}(z)$.

We will need later the following version of the same principle:

Lemma 3.11. Let $S$ be a split locus, and $h$ be the function associated to $S$ as in definition 2.4. If $\rho_{S}$ is continuous, and $h$ can be extended to $\Omega$ so that it is continuous except for a set of null $\mathcal{H}^{n-1}$ measure, then $S=$ Sing.

Proof. Define

$$
Y_{0}=\left\{z \in \partial \Omega: h(F(t, z)) \neq u(F(t, z)) \text { for some } t \in\left[0, \rho_{\text {Sing }}(z)\right)\right\}
$$

By the claim in the previous lemma, $Y_{0}$ is contained in:

$$
Y=\left\{z \in \partial \Omega: h \text { discontinuous at } F(t, z) \text { for some } t \in\left[0, \rho_{\text {Sing }}(z)\right)\right\}
$$


Let $A=A($ Sing $)$ be the set in definition 2.1. The map $F$ restricts to a diffeomorphism from $A$ onto $\Omega \backslash$ Sing. The set $Y$ can be expressed as:

$$
\left.Y=\pi_{2} \circ\left(\left.F\right|_{A}\right)^{-1}(\{p \in \Omega \backslash \text { Sing : } h \text { discontinuous at } p\})\right)
$$

and thus by the hypothesis has null $\mathcal{H}^{n-1}$ measure. Therefore, $\partial \Omega \backslash Y_{0}$ is dense in $\partial \Omega$.

We claim now that $S \subset$ Sing. To see this, let $p \in S \backslash$ Sing. Then $p=F\left(t^{*}, z^{*}\right)$ for a unique $\left(t^{*}, z^{*}\right) \in A$. It follows $\rho_{S}\left(z^{*}\right) \leq t^{*}<\rho_{\text {Sing }}\left(z^{*}\right)$. As $\rho_{S}$ is continuous, $\rho_{S}(z)<\rho_{\text {Sing }}(z)$ holds for all $z$ in a neighborhood of $z^{*}$ in $\partial \Omega$ and, in particular, for some $z \in \partial \Omega \backslash Y_{0}$. This is a contradiction because, for $\rho_{S}(z)<t<\rho_{\text {Sing }}(z), h(F(t, z))=\tilde{u}\left(t^{\prime}, z^{\prime}\right)$ for $\left(t^{\prime}, z^{\prime}\right) \neq(t, z)$, and $t<\rho_{\text {Sing }}(z)$ implies $h(F(t, z))=\tilde{u}\left(t^{\prime}, z^{\prime}\right)>\tilde{u}(t, z)=u(F(t, z))$, forcing $z \in Y_{0}$.

We deduce $S=$ Sing using lemma 2.6 and the fact that Sing is a split locus.

\section{$4 \rho_{S}$ is Lipschitz}

In this section we study the functions $\rho_{S}$ and $\lambda_{j}$ defined earlier. The fact that $\rho_{S}$ is Lipschitz will be of great importance later. The definitions and the general approach in this section follow [IT], but our proofs are shorter, provide no precise quantitative bounds, use no constructions from Riemannian or Finsler geometry, and work for Finsler manifolds, thus providing a new and shorter proof for the main result in [LN]. The proof that $\lambda_{j}$ are Lipschitz functions was new for Finsler manifolds when we published the first version of the preprint of this paper. Since then, another preprint has appeared which shows that $\lambda_{1}$ is actually semi-concave.

Proof of 2.13. It is immediate to see that the functions $\lambda_{j}$ are continuous, since this is property (R3) of Warner (see [W, pp. 577-578 and Theorem 4.5 ]).

Near a conjugate point $x^{0}$ of order $k$, we can take special coordinates as in 3.2 .

$$
F\left(x_{1}, \ldots, x_{n}\right)=\left(x_{1}, \ldots, x_{n-k}, F_{n-k+1}, \ldots, F_{n}\right)
$$

Conjugate points near $x$ are the solutions of

$$
d\left(x_{1}, \ldots, x_{n}\right)=\operatorname{det}(d F)=\sum_{\sigma}(-1)^{\sigma} \frac{\partial F_{\sigma(n-k+1)}}{\partial x_{n-k+1}} \ldots \frac{\partial F_{\sigma(n)}}{\partial x_{n}}=0
$$

$>$ From the properties of the special coordinates, we deduce that:

$$
D^{\alpha} d(0)=0 \quad \forall|\alpha|<k
$$

and

$$
\frac{\partial^{k}}{\partial x_{1}^{k}} d=1
$$

We can use the preparation theorem of Malgrange (see [GG]) to find real valued functions $q$ and $l_{i}$ in an open neighborhood $U$ of $x$ such that $q(x) \neq 0$ and:

$$
q\left(x_{1}, \ldots, x_{n}\right) d\left(x_{1}, \ldots, x_{n}\right)=x_{1}^{k}+x_{1}^{k-1} l_{1}\left(x_{2}, \ldots, x_{n}\right)+\cdots+l_{k}\left(x_{2}, \ldots, x_{n}\right)
$$

and we deduce from (4.1) that

$$
D^{\alpha} l_{i}(0)=0 \quad \forall|\alpha|<i
$$


which implies

$$
\left|l_{i}\left(x_{2}, \ldots, x_{n}\right)\right|<\bar{C} \max \left\{\left|x_{2}\right|, \ldots,\left|x_{n}\right|\right\}^{i}
$$

At any conjugate point $\left(x_{1}, \ldots, x_{n}\right)$, we have $q(x)=0$, so:

$$
-x_{1}^{k}=x_{1}^{k-1} l_{1}\left(x_{2}, \ldots, x_{n}\right)+\cdots+l_{k}\left(x_{2}, \ldots, x_{n}\right)
$$

and therefore

$$
\left|x_{1}\right|^{k}<\left|x_{1}\right|^{k-1}\left|l_{1}\right|+\cdots+\left|l_{k}\right|
$$

Combining this and (4.3), we get an inequality for $\left|x_{1}\right|$ at any conjugate point $\left(x_{1}, \ldots, x_{n}\right)$, where the constant $C$ ultimately depends on bounds for the first few derivatives of $F$ :

$$
\left|x_{1}\right|^{k}<C \max \left\{\left|x_{1}\right|, \ldots,\left|x_{n}\right|\right\}^{k-1} \max \left\{\left|x_{2}\right|, \ldots,\left|x_{n}\right|\right\}
$$

We notice that $\left|x_{1}\right|>\max \left\{\left|x_{2}\right|, \ldots,\left|x_{n}\right|\right\}$ implies $\left|x_{1}\right|^{k}<C\left|x_{1}\right|^{k-1} \max \left\{\left|x_{2}\right|, \ldots,\left|x_{n}\right|\right\}$. In other words:

$$
\left|x_{1}\right|<\max \{C, 1\} \max \left\{\left|x_{2}\right|, \ldots,\left|x_{n}\right|\right\}
$$

This is the statement that all conjugate points near $x$ lie in a cone of fixed width containing the hyperplane $x_{1}=0$. Thus all functions $\lambda_{j}$ to $\lambda_{j+k}$ are Lipschitz at $\left(x_{2} \ldots, x_{n}\right)$ with a constant independent of $x$.

Remark. A proof of lemma 2.13 in the lines of section 3.3 seems possible: let $\Lambda(\Omega)$ be the bundle of Lagrangian submanifolds of the symplectic linear spaces $T_{p}^{*} \Omega$ and let $\Sigma(\Omega)$ be the union of the Maslov cycles within each $\Lambda_{p}(\Omega)$. Define $\lambda: V \rightarrow \Lambda(\Omega)$ where $\lambda(x)$ is the tangent to $\Theta$ at $D\left(\Phi(x)\right.$ ) (recall 3.5). The graphs of the functions $\lambda_{k}$ are the preimage of the Maslov cycle $\Sigma(\Omega)$. The geodesic vector field (transported to $T^{*} \Omega$ ), is transversal to the Maslov cycle. With some effort, the angle (in an arbitrary metric) between this vector field and the Maslov cycle at points of intersection can be bounded from below. This is sufficient to show that the $\lambda_{k}$ are Lipschitz.

Lemma 4.1. For any split locus $S$ and point $y \in \partial \Omega$, there are no conjugate points in the curve $t \rightarrow \exp (t y)$ for $t<\rho_{S}(y)$. In other words, $\rho_{S} \leq \lambda_{1}$.

Proof. Assume there is $x$ with $\rho_{S}(x)-\varepsilon>\lambda_{1}(x)$. By [W] 3.4], the map $F$ is not injective in any neighborhood of $(x, t)$. There are points $\left(x_{n}, t_{n}\right)$ of $S$ with $x_{n} \rightarrow x$ and $t_{n}<\rho_{S}(x)-\varepsilon$ (otherwise $S$ does not split $\Omega$ ). Taking limits, we see $F(x, t)$ is in $S$ for some $t<\rho_{S}(x)-\varepsilon$, which contradicts the definition of $\rho_{S}(x)$.

$>$ From now on and for the rest of the paper, $S$ will always be a balanced split locus:

Lemma 4.2. Let $E \subset \partial \Omega$ be an open subset whose closure is compact and has a neighborhood where $\rho<\lambda_{1}$. Then $\rho_{S}$ is Lipschitz in E.

Proof. The map $x \rightarrow\left(F(x), d F_{x}(r)\right)$ is an embedding of $V$ into $T M$. There is a constant $c$ such that for $x, y \in V$ :

$$
|F(x)-F(y)|+\left|d F_{x}(r)-d F_{y}(r)\right| \geq c \min \{|x-y|, 1\}
$$

Recall the exponential map is a local diffeomorphism before the first conjugate point. Points $p=F((z, \rho(z)))$ for $z \in E$ have a set $R_{p}$ consisting of the vector $d F_{(z, \rho(z))}(r)$, and vectors coming 
from $V \backslash E$. Choose one such point $p$, and a neighborhood $U$ of $p$. The above inequality shows that there is a constant $m$ such that:

$$
\left|d F_{x}(r)-d F_{y}(r)\right| \geq m
$$

for $x=(z, \rho(z))$ with $z \in E$ and $y=(w, \rho(w)) \in Q_{p}$ with $w \in V \backslash E$. By the balanced condition 2.7, any unit vector $v$ tangent to $S$ satisfies $\widehat{d F_{x}(r)}(v)=\widehat{d F_{y}(r)}(v)$ for some such $y$ and so:

$$
\widehat{d F_{x}(r)}(v)<1-\varepsilon
$$

Thus for any vector $w$ tangent to $E$ both vectors $\left(w, d \rho_{-}(w)\right)$ and $\left(w, d \rho_{+}(w)\right)$ lie in a cone of fixed amplitude around the kernel of $\widehat{d F_{x}(r)}$ (the hyperplane tangent to the indicatrix at $x$ ). Application of lemma 3.7 shows that $\rho$ is Lipschitz.

Lemma 4.3. Let $z_{0} \in \partial \Omega$ be a point such that $\rho\left(z_{0}\right)=\lambda_{1}\left(z_{0}\right)$. Then there is a neighborhood $E$ of $z_{0}$ and a constant $C$ such that for all $z$ in $E$ with $\rho(z)<\lambda_{1}(z), \rho$ is Lipschitz near $z$ with Lipschitz constant $C$.

Proof. Let $O$ be a compact neighborhood of $\left(z_{0}, \lambda_{1}\left(z_{0}\right)\right)$ where special coordinates apply. Let $x=(z, \rho(z)) \in O$ be such that $\rho(z)<\lambda_{1}(z)$. We can apply the previous lemma and find $\rho$ is Lipschitz near $z$. We just need to estimate the Lipschitz constant uniformly. Vectors in $R_{F(x)}$ that are of the form $d F_{y}(r)$ for $y \in V \backslash O$, are separated from $d F_{x}(r)$ as in the previous lemma and pose no trouble, but now there might be other vectors $d F_{y}(r)$ for $y \in O$.

Fix the metric $\langle\cdot\rangle$ in $O$ whose matrix in special coordinates is the identity. Any tangent vector to $S$ satisfies $\widehat{d F_{x}(r)}(v)=\widehat{d F_{y}(r)}(v)$, for some $y \in O \cap Q_{F(x)}$. A uniform Lipschitz constant for $\rho$ is found if we bound from below the angle in the metric $\langle\cdot\rangle$ between $r$ and $d_{x} F^{-1}(v)$ for any vector $v$ with this property. Fix a point $y \in O$ with $F(x)=F(y)$, let $X=d F_{x}(r), Y=d F_{y}(r)$ and $\alpha=\widehat{X}-\widehat{Y}$. We need to bound from below the angle between $r$ and the hyperplane ker $\alpha$.

Our goal is equivalent to proving that there is $\varepsilon_{1}>0$ independent of $x$ such that:

$$
\frac{F_{x}^{*} \alpha(r)}{\left\|F_{x}^{*} \alpha\right\|}>\varepsilon_{1}
$$

which is equivalent to:

$$
\widehat{Y}(X)<1-\varepsilon_{1}\left\|F_{x}^{*} \alpha\right\|
$$

in the norm $\|\cdot\|$ associated to $\langle\cdot\rangle$.

Notice first that $X$ and $Y$ belong to the indicatrix at $F(x)=F(y)$, which is strictly convex. By this and (4.5), we see that for some $\varepsilon_{2}>0$ :

$$
\widehat{Y}(X)<1-\varepsilon_{2}\|X-Y\|^{2}<1-c \varepsilon_{2}\|x-y\|^{2}
$$

So it is sufficient to show that for some $C_{1}$ independent of $x$ :

$$
\left\|F_{x}^{*} \alpha\right\|<C_{1}\|x-y\|^{2}
$$

Using a Taylor expansion of $\frac{\partial \varphi}{\partial x_{j}}$ in the second entry, we see the form $F_{x}^{*} \alpha$ can be written in coordinates:

$$
\begin{aligned}
F_{x}^{*} \alpha & =\left(\frac{\partial \varphi}{\partial x_{j}}(p, X)-\frac{\partial \varphi}{\partial x_{j}}(p, Y)\right) \frac{\partial F_{j}}{\partial x_{l}} \\
& =\frac{\partial^{2} \varphi}{\partial x_{i} x_{j}}(p, X)\left(X_{i}-Y_{i}\right) \frac{\partial F_{j}}{\partial x_{l}}+O(\|X-Y\|)^{2} \\
& =\frac{\partial^{2} \varphi}{\partial x_{i} x_{j}}(p, X)\left(X_{i}-Y_{i}\right) \frac{\partial F_{j}}{\partial x_{l}}+O(\|x-y\|)^{2}
\end{aligned}
$$


The argument goes as follows: we need the inequality $\left\|F_{x}^{*} \alpha(v)\right\|<C_{1}\|v\|\|x-y\|^{2}$, so we want to bound the bilinear map $g_{(p, X)}$ evaluated at $X-Y$ and the vector $d F(v)$. The bound on the norm is achieved when $d F(v)$ is proportional to $X-Y$. The map $d_{x} F$ is invertible, so for the vector $v=\frac{d F^{-1}(X-Y)}{\left\|d F^{-1}(X-Y)\right\|}$, we have:

$$
\left\|F_{x}^{*} \alpha\right\|=\left\|F_{x}^{*} \alpha(v)\right\|
$$

Thus we have:

$$
\left\|F_{(z, \rho(z))}^{*} \alpha\right\|<C_{2} \frac{\|X-Y\|^{2}}{\left\|d F^{-1}(X-Y)\right\|}+O(\|x-y\|)^{2}<C_{3} \frac{\|x-y\|^{2}}{\left\|d F^{-1}(X-Y)\right\|}+O(\|x-y\|)^{2}
$$

for constants $C_{2}$ and $C_{3}$, and it is enough to show there is $\varepsilon_{3}$ independent of $x$ and $y$ such that:

$$
\left\|d F^{-1}(X-Y)\right\|>\varepsilon_{3}
$$

Let $G(x)=d_{x} F(r)$. We have:

$$
X-Y=G(x)-G(y)=d G_{x}(x-y)+O(\|x-y\|)
$$

so it is equivalent to show the following:

$$
\left\|d F^{-1} d G_{x}(x-y)\right\|>\varepsilon_{4}
$$

for $\varepsilon_{4}$ independent of $x$ and $y$.

Assume that $\left(\rho\left(z_{0}\right), z_{0}\right)$ is conjugate of order $k$, so that $\rho\left(z_{0}\right)=\lambda_{1}\left(z_{0}\right)=\cdots=\lambda_{k}\left(z_{0}\right)$. Thanks to Lemma 2.13 and reducing to a smaller $O$, we can assume that $a_{1}=\left(\lambda_{1}(z), z\right)$ to $a_{k}=\left(\lambda_{k}(z), z\right)$ all lie within $O$ (some of them may coincide). Let $d_{i}=\lambda_{i}(z)-\rho(z)$ be the distance from $x$ to the $a_{i}$. At each of the $a_{i}$ there is a vector $w_{i} \in \operatorname{ker} d_{a_{i}} F$ such that all the $w_{i}$ span a $k$-dimensional subspace. Recall from section 3.3 that we can choose $w_{i}$ forming an almost orthonormal subset for the above metric, in the sense that $\left\langle w_{i}, w_{j}\right\rangle=\delta_{i, j}+\varepsilon_{i, j}$.

The kernel of $d_{y} F$ is contained in $K=\left\langle\frac{\partial}{\partial x_{n-k+1}}, \ldots, \frac{\partial}{\partial x_{n}}\right\rangle$ for all $y \in O$, and thus $K=$ $\left\langle w_{1}, \ldots, w_{k}\right\rangle$. Write $w_{i}=\sum_{j \geq n-k+1} w_{i}^{j} \frac{\partial}{\partial x^{j}}$. Then we have $\frac{\partial}{\partial x^{1}} \frac{\partial}{\partial w_{i}} F(a)=z_{i}+R_{i}(a)$, for $z_{i}=$ $\sum w_{i}^{k} \frac{\partial}{\partial y^{k}},\left\|R_{i}(a)\right\|<\varepsilon$ and $a \in O$. We deduce $\frac{\partial}{\partial w_{i}} F(x)=\frac{\partial}{\partial w_{i}} F\left(a_{i}\right)+d_{i}\left(z_{i}+v_{i}\right)=d_{i}\left(z_{i}+v_{i}\right)$ for $\left\|v_{i}\right\|<\varepsilon$.

By the form of the special coordinates, $x-y \in K$. Let $x-y=\sum b_{i} w_{i}$. Since $\left|w_{i}\right|$ is almost 1 , there is an index $i_{0}$ such that $\left|b_{i_{0}}\right|>\frac{1}{2 n}\|x-y\|$. We have the identity:

$$
0=F(y)-F(x)=d_{x} F(y-x)+O\left(\|x-y\|^{2}\right)=\sum b_{i} d_{i}\left(z_{i}+v_{i}\right)+O\left(\|x-y\|^{2}\right)
$$

Multiplying the above by $\pm z_{j}$, we deduce $d_{j}\left|b_{j}\right|=-\sum\left|b_{i}\right| d_{j}\left(\varepsilon_{i, j}+v_{i} z_{j}\right)+O\left(\|x-y\|^{2}\right)$, which leads to

$$
\sum\left|b_{i}\right| d_{i}<C_{4}\|x-y\|^{2}
$$

At the point $x$, the image by $d_{x} F$ of the unit ball $B_{x} V$ in $T_{x} V$ is contained in a neighborhood of $\operatorname{Im}\left(d_{a_{i}} F\right)$ of radius $2 d_{i}$. We use the identity

$$
\left\|d F^{-1} d G_{x}\left(\frac{x-y}{\|x-y\|}\right)\right\|^{-1}=\sup \left\{t: t d G_{x}\left(\frac{x-y}{\|x-y\|}\right) \in d_{x} F\left(B_{x} V\right)\right\}
$$


We can assume the distance between the vectors $d G_{x}\left(\frac{x-y}{\|x-y\|}\right)$ and $\sum \frac{b_{i}}{\|x-y\|} z_{i}$ is smaller than $\frac{1}{4 n}$. In particular, looking at the $i_{0}$ coordinate chosen above, we see that the vector $d G_{x}\left(\frac{x-y}{\|x-y\|}\right)$ needs to be rescaled at least by the amount $8 n d_{i}$ in order to fit within the image of the unit ball.

$$
\left\|d F^{-1} d G_{x}\left(\frac{x-y}{\|x-y\|}\right)\right\|>\frac{1}{8 n d_{i_{0}}}>\frac{\left|b_{i_{0}}\right|}{8 n C_{4}\|x-y\|^{2}}>\frac{\varepsilon_{4}}{\|x-y\|}
$$

for $\varepsilon_{4}=\frac{1}{16 n^{2} C_{4}}>0$, which is the desired inequality.

Proof of Lemma 2.14. We prove that $\rho$ is Lipschitz close to a point $z^{0}$. Let $E$ be a neighborhood of $z^{0}$ such that $\lambda_{1}$ has Lipschitz constant $L$, and $\rho$ has Lipschitz constant $K$ for all $z \in E$ such that $\rho(z)<\lambda(z)$. Let $z^{1}, z^{2} \in E$ be such that $\rho\left(z^{1}\right)<\rho\left(z^{2}\right)$.

If $\rho\left(z^{1}\right)=\lambda_{1}\left(z^{1}\right)$ we can compute

$$
\left|\rho\left(z^{2}\right)-\rho\left(z^{1}\right)\right|=\rho\left(z^{2}\right)-\rho\left(z^{1}\right)<\lambda\left(z^{2}\right)-\lambda\left(z^{1}\right)<L\left|z^{2}-z^{1}\right|
$$

where $L$ is a Lipschitz constant $L$ for $\lambda$ in $U$.

Otherwise take a linear path with unit speed $\xi:[0, t] \rightarrow \partial \Omega$ from $z^{1}$ to $z^{2}$ and let $a$ be the supremum of all $s$ such that $\rho(\xi(s))<\lambda(\xi(s))$. Then

$$
\left|\rho\left(z^{2}\right)-\rho\left(z^{1}\right)\right|<\left|\rho\left(z^{2}\right)-\rho(\xi(a))\right|+\left|\rho(\xi(a))-\rho\left(z^{1}\right)\right|
$$

The second term can be bound:

$$
\left|\rho(\xi(a))-\rho\left(z^{1}\right)\right|<K a
$$

If $\rho\left(z^{2}\right) \geq \rho(\xi(a))$, we can bound the first term as

$$
\left|\rho\left(z^{2}\right)-\rho(\xi(a))\right|=\rho\left(z^{2}\right)-\rho(\xi(a))<\lambda\left(z_{2}\right)-\lambda(\xi(a))<L|t-a|
$$

while if $\rho\left(z^{2}\right)<\rho(\xi(a))$, we have

$$
\left|\rho\left(z^{2}\right)-\rho\left(z^{1}\right)\right|<\left|\rho(\xi(a))-\rho\left(z^{1}\right)\right|
$$

so in all cases, the following holds:

$$
\left|\rho\left(z^{2}\right)-\rho\left(z^{1}\right)\right|<\max \{L, K\} t<\max \{L, K\}\left|z^{2}-z^{1}\right|
$$

\section{Proof of the main theorems.}

Take the function $h$ associated to $S$ as in definition 2.4. At a cleave point $x$ there are two geodesics arriving from $\partial \Omega$; each one yields a value of $h$ by evaluation of $\tilde{u}$. The balanced condition implies that $\widehat{X}_{1}(v)=\widehat{X}_{2}(v)$ for the speed vectors $X_{1}$ and $X_{2}$ of the characteristics reaching $x$ and any vector $v$ tangent to $S$. Furthermore, $\widehat{X}$ is exactly $d h$, so the difference of the values of $h$ from either side is constant in every connected component of the cleave locus.

We define an $(n-1)$-current $T$ in this way: Fix an orientation $\mathcal{O}$ in $\Omega$. For every smooth $(n-1)$ differential form $\phi$, restrict it to the set of cleave points $\mathcal{C}$ (including degenerate cleave points). In every component $\mathcal{C}_{j}$ of $\mathcal{C}$ compute the following integrals 


$$
\int_{\mathcal{C}_{j, i}} h_{i} \phi \quad i=1,2
$$

where $\mathcal{C}_{j, i}$ is the component $\mathcal{C}_{j}$ with the orientation induced by $\mathcal{O}$ and the incoming vector $V_{i}$, and $h_{i}$ for $i=1,2$ are the limit values of $h$ from each side of $\mathcal{C}_{j}$.

We define the current $T(\phi)$ to be the sum:

$$
T(\phi)=\sum_{j} \int_{\mathcal{C}_{j, 1}} h_{1} \phi+\int_{\mathcal{C}_{j, 2}} h_{2} \phi=\sum_{j} \int_{\mathcal{C}_{j, 1}}\left(h_{1}-h_{2}\right) \phi
$$

The function $h$ is bounded and the $\mathcal{H}^{n-1}$ measure of $\mathcal{C}$ is finite (thanks to lemma 2.14) so that $T$ is a real flat current that represents integrals of test functions against the difference between the values of $h$ from both sides.

If $T=0$, we can apply lemma 3.11 and find $u=h$.

We will prove later that the boundary of $T$ as a current is zero. Assume for the moment that $\partial T=0$. It defines an element of the homology space $H_{n-1}(\Omega)$ of dimension $n-1$ with real coefficients. We can study this space using the long exact sequence of homology with real coefficients for the pair $(\Omega, \partial \Omega)$ :

$$
\begin{array}{r}
0 \rightarrow H_{n}(\Omega) \rightarrow H_{n}(\Omega, \partial \Omega) \rightarrow \\
H_{n-1}(\partial \Omega) \rightarrow H_{n-1}(\Omega) \rightarrow H_{n-1}(\Omega, \partial \Omega) \rightarrow \ldots
\end{array}
$$

\subsection{Proof of Theorem 2.9.}

We prove that under the hypothesis of 2.9 , the space $H_{n-1}(\Omega)$ is zero, and then we deduce that $T=0$.

As $\Omega$ is open, $H_{n}(\Omega) \approx 0$. As $\Omega$ is simply connected, it is orientable, so we can apply Lefschetz duality with real coefficients ([Ha, 3.43]) which implies:

$$
H_{n}(\Omega, \partial \Omega) \approx H^{0}(\Omega)
$$

and

$$
H_{n-1}(\Omega, \partial \Omega) \approx H^{1}(\Omega)=0
$$

As $\partial \Omega$ is connected, we deduce $H_{n-1}(\Omega)$ has rank 0 , and $T=\partial P$ for some $n$-dimensional flat current $P$. The flat top-dimensional current $P$ can be represented by a density $f \in L^{n}(\Omega)$ (see $[\mathrm{F}$, p 376, 4.1.18]):

$$
P(\omega)=\int_{\Omega} f \omega,, \quad \omega \in \Lambda^{n}(\Omega)
$$

We deduce from (5.2) that the restriction of $P$ to any open set disjoint with $S$ is closed, so $f$ is a constant in such open set. As $\Omega \backslash S$ is open and connected, $f$ is constant a.e., and $T=0$.

\subsection{Proof of Theorem 2.10 .}

Assume now that $\partial \Omega$ has $k$ connected components $\Gamma_{i}$. We look at (5.3), and recall the map $H_{n-1}(\partial \Omega) \rightarrow H_{n-1}(\Omega)$ is induced by inclusion. We know by Poincaré duality that $H_{n-1}(\partial \Omega)$ is isomorphic to the linear combinations of the fundamental classes of the connected components of 
$\partial \Omega$ with real coefficients. We deduce that $H_{n-1}(\Omega)$ is generated by the fundamental classes of the connected components of $\partial \Omega$, and that it is isomorphic to the quotient of all linear combinations by the subspace of those linear combinations with equal coefficients. Let

$$
R=\sum a_{i}\left[\Gamma_{i}\right]
$$

be the cycle to which $T$ is homologous (the orientation of $\Gamma_{i}$ is such that, together with the inwards pointing vector, yields the ambient orientation).

If we define $a(x)=a_{i}, \forall x \in \Gamma_{i}$, solve the HJ equations with boundary data $g-a$ and compute the corresponding current $\widehat{T}$, we see that $\widehat{T}=T-j_{\sharp} R$, where $j$ is the retraction $j$ of $\Omega$ onto $S$ that fixes points of $S$ and follows characteristics otherwise. Then the homology class of $\widehat{T}$ is zero, and we can prove $\widehat{T}=0$ as before. It follows that $S$ is the singular set to the solution of the Hamilton-Jacobi equations with boundary data $g-a$.

\subsection{Proof of Theorem 2.12 .}

For this result we cannot simply use the sequence (5.3). We first give a procedure for obtaining balanced split loci in $\Omega$ other than the cut locus.

A function $a:[\partial \widetilde{\Omega}] \rightarrow \mathbb{R}$ that assigns a real number to each connected component of $\partial \widetilde{\Omega}$ is equivariant iff for any automorphism of the cover $\varphi$ there is a real number $c(\varphi)$ such that $a \circ \varphi=a+c(\varphi)$.

A function $a:[\partial \widetilde{\Omega}] \rightarrow \mathbb{R}$ is compatible iff $\widetilde{g}-a$ satisfies the compatibility condition (1.3).

An equivariant function $a$ yields a group homomorphism from $\pi_{1}(\Omega, \partial \Omega)$ into $\mathbb{R}$ in this way:

$$
\sigma \rightarrow a(\widetilde{\sigma}(1))-a(\widetilde{\sigma}(0))
$$

where $\sigma:[0,1] \rightarrow \Omega$ is a path with endpoints in $\partial \Omega$ and $\widetilde{\sigma}$ is any lift to $\widetilde{\Omega}$. The result is independent of the lift because $a$ is equivariant. On the other hand, choosing an arbitrary component $\left[\Gamma_{0}\right]$ of $\partial \Omega$ and a constant $a_{0}=a([\Gamma])$, the formula:

$$
[\Gamma] \rightarrow a\left(\left[\Gamma_{0}\right]\right)+l(\pi \circ \tilde{\sigma}), \text { for any path } \tilde{\sigma} \text { with } \tilde{\sigma}(0) \in \Gamma_{0}, \sigma(1) \in \Gamma
$$

assigns an equivariant function $a$ to an element $l$ of $H o m\left(\pi_{1}(\Omega, \partial \Omega), \mathbb{R}\right) \sim H^{1}(\Omega, \partial \Omega)$.

Up to addition of a global constant, these two maps are inverse of one another, so there is a one-to-one correspondence between elements of $H^{1}(\Omega, \partial \Omega)$ and equivariant functions $a$ (with $a+c$ identified with $a$ for any constant $c$ ). The compatible equivariant functions up to addition of a global constant can be identified with an open subset of $H^{1}(\Omega, \partial \Omega)$ that contains the zero cohomology class.

Let $\widetilde{\Omega}$ be the universal cover of $\Omega$. We can lift the Hamiltonian $H$ to a function $\widetilde{H}$ defined on $T^{*} \widetilde{\Omega}$ and the function $g$ to a function $\tilde{g}$ defined on $\partial \widetilde{\Omega}$. The preimage of a balanced split locus for $\Omega, H$ and $g$ is a balanced split locus for $\widetilde{\Omega}, \widetilde{H}$ and $\tilde{g}$ that is invariant by the automorphism group of the cover, and conversely, a balanced split locus $\widetilde{S}$ in $\widetilde{\Omega}$ that is invariant by the automorphism group of the cover descends to a balanced split locus on $\Omega$.

Any function $a$ that is both equivariant and compatible can be used to solve the HamiltonJacobi problem $\widetilde{H}(p, d u(p))=1$ in $\widetilde{\Omega}$ and $u(p)=\widetilde{g}(p)-a(p)$. If $\pi_{1}(\Omega)$ is not finite, $\widetilde{\Omega}$ will not be compact, but this is not a problem (see remark 5.5 in page 125 of $[\mathrm{L}]$ ). The singular set is a balanced split locus that is invariant under the action of $\pi_{1}(\Omega)$ and hence it yields a balanced split locus in $\Omega$. We write $S[a]$ for this set. It is not hard to see that the map $a \rightarrow S[a]$ is injective. 
Conversely, a balanced split locus in $\Omega$ lifts to a balanced split locus $\widetilde{S}$ in $\widetilde{\Omega}$. The reader may check that the current $T_{\widetilde{S}}$ is the lift of $T_{S}$, and in particular it is closed. As in the proof of Theorem 2.10, we have $H^{1}(\widetilde{\Omega})=0$, and we deduce

$$
T_{\widetilde{S}}=\sum_{j} a_{j}\left[\Lambda_{j}\right]+\partial P
$$

where $\Lambda_{j}$ are the connected components of $\partial \widetilde{\Omega}$.

This class is the lift of the class of $T \in H_{n-1}(\Omega)$ and thus it is invariant under the action of the group of automorphisms of the cover. Equivalently, the map defined in (5.5) is a homomorphism. Thus $a$ is equivariant. Similar arguments as before show that $S=S[a]$.

Thus the map $a \rightarrow S[a]$ is also surjective, which completes the proof that there is a bijection between equivariant compatible functions $a:[\partial \widetilde{\Omega}] \rightarrow \mathbb{R}$ and balanced split loci.

\section{Proof that $\partial T=0$}

It is enough to show that $\partial T=0$ at all points of $\Omega$ except for a set of zero $(n-2)$-dimensional Hausdorff measure. This is clear for points not in $S$. Due to the structure result [3.5, we need to show the same at cleave points (including degenerate ones), edge points and crossing points. Along the proof, we will learn more about the structure of $S$ near those kinds of points.

Throughout this section, we assume $n=\operatorname{dim}(\Omega)>2$. This is only to simplify notation, but the case $n=2$ is covered too. We shall comment on the necessary changes to cover the case $n=2$, but do not bother with the simple case $n=1$.

\subsection{Conjugate points of order 1 .}

We now take a closer look at points of $A(S)$ that are also conjugate points of order 1 . Fortunately, because of 3.5 we do not need to deal with higher order conjugate points. In a neighborhood $O$ of a point $x^{0}$ of order 1 , in the special coordinates of section $\underline{3.2}$, we have $x^{0}=0$ and $F$ looks like:

$$
F\left(x_{1}, x_{2}, \ldots, x_{n}\right)=\left(x_{1}, x_{2}, \ldots, F_{n}\left(x_{1}, \ldots, x_{n}\right)\right)
$$

Let $\widetilde{S}$ be the boundary of $A(S)$, but without the points $(0, z)$ for $z \in \partial \Omega$. It follows from 2.14 that $\widetilde{S}$ is a Lipschitz graph on coordinates given by the vector field $r$ and $n-1$ transversal coordinates. It is not hard to see that it is also a Lipschitz graph $x_{1}=\tilde{t}\left(x_{2}, \ldots, x_{n}\right)$ in the above coordinates $x_{i}$, possibly after restricting to a smaller open set.

Because of Lemma 4.1, we know $x^{0}$ is a first conjugate point, so we can assume that $O$ is a coordinate cube $\prod\left(-\varepsilon_{i}, \varepsilon_{i}\right)$, and that $F$ is a diffeomorphism when restricted to $\left\{x_{1}=s\right\}$ for $s<-\varepsilon_{1} / 2$.

Definition 6.1. A set $O \subset V$ is univocal iff for any $p \in \Omega$ and $x^{1}, x^{2} \in Q_{p} \cap O$ we have $\tilde{u}\left(x^{1}\right)=\tilde{u}\left(x^{2}\right)$.

Remark. The most common case of univocal set is a set $O$ such that $F \mid O$ is injective.

Lemma 6.2. Let $x^{0} \in V$ be a conjugate point of order 1 . Then $x^{0}$ has an univocal neighborhood. 
Proof. Let $O_{1}$ and $U_{1}$ be neighborhoods of $x^{0}$ and $F\left(x^{0}\right)$ where the special coordinates (6.1) hold; let $x_{i}$ be the coordinates in $O_{1}$ and $y_{i}$ be those in $U_{1}$.

Choose smaller $U \subset U_{1}$ and $O \subset F^{-1}(U) \cap O_{1}$ so that we can assume that if a point $x^{\prime} \in V \backslash O_{1}$ maps to a point in $U$, then for the vector $Z=d F_{x^{\prime}}(r)$ we have

$$
\hat{Z}\left(\frac{\partial}{\partial y_{1}}\right)<\hat{X}\left(\frac{\partial}{\partial y_{1}}\right)
$$

for any $X=d F_{x}(r)$ with $x \in O$ and also

$$
\hat{Y}\left(\frac{\partial}{\partial y_{1}}\right)>1-k
$$

for some $k>0$ sufficiently small and all $Y=d F_{y^{\prime}}(r)$ for $y^{\prime} \in O_{1}$.

Take $x^{1}, x^{2} \in Q_{q} \cap O$ for $q \in U$. The hypothesis $x^{1}, x^{2} \in Q_{q}$ implies $q=F\left(x^{1}\right)=F\left(x^{2}\right)$, and so $x_{j}^{1}=x_{j}^{2}$ follows for all $j<n$. Let us write $a_{j}=x_{j}^{1}=x_{j}^{2}$ for $j<n, s^{1}=x_{n}^{1}$ and $s^{2}=x_{n}^{2}$. Fix $a_{2}, \ldots, a_{n-1}$ and consider the set

$$
H_{a}=\left\{x \in O: x_{i}=a_{i} ; i=2, \ldots, n-1\right\}
$$

Its image by $F$ is a subset of a plane in the $y_{i}$ coordinates:

$$
L_{a}=\left\{y \in U: y_{i}=a_{i}, i=2, \ldots, n-1\right\}
$$

Points of $O_{1}$ not in $H_{a}$ map to other planes. If $n=2$, we keep the same notation, but the meaning is that $H_{a}=O$ and $L_{a}=V$.

There is $\varepsilon>0$ such that for $t<-\varepsilon / 2$, the line $\left\{x_{1}=t\right\} \cap H_{a}$ maps diffeomorphically to $\left\{y_{1}=t\right\} \cap L_{a}$.

Due to the comments at the beginning of this section, $\widetilde{S}$ is given as a Lipschitz graph $x_{1}=$ $\tilde{t}\left(x_{2}, \ldots, x_{n}\right)$. The identity $a_{1}=\tilde{t}\left(a_{2}, \ldots, a_{n-1}, s^{i}\right)$ holds for $i=1,2$ because $x^{1}, x^{2} \in Q_{q}$. We define a curve $\sigma:\left[s^{1}, s^{2}\right] \rightarrow \widetilde{S}$ by $\sigma(s)=\left(\tilde{t}\left(a_{2}, \ldots, a_{n-1}, s\right), a_{2}, \ldots, a_{n-1}, s\right)$. The image of $\sigma$ by $F$ stays in $S$, describing a closed loop based at $q$; we will establish the lemma by examining the variation of $\tilde{u}$ along $\sigma$.

For $i=1,2$, let $\eta^{i}:\left(-\varepsilon_{i}, a_{1}\right] \rightarrow H_{a}$ given by $\eta^{i}(t)=\left(t, a_{2}, \ldots, a_{n-1}, s^{i}\right)$ be the segments parallel to the $x_{1}$ direction that end at $x^{i}$, defined from the first point in the segment that is in $O$. We can assume that the intersection of $O$ with any line parallel to $\frac{\partial}{\partial x_{1}}$ is connected, and that the intersection of $U$ with any line parallel to $\frac{\partial}{\partial y_{1}}$ is connected too. We can also assume $\varepsilon_{i}<\varepsilon$.

Let $D$ be the closed subset of $H_{a}$ delimited by the Lipschitz curves $\eta^{1}, \eta^{2}$ and $\sigma$, and let $E$ be the closed subset of $L_{a}$ delimited by the image of $\eta^{1}$ and $\eta^{2}$.

We claim $D$ is mapped onto $E$. First, no point in $\operatorname{int}(D)$ can map to the image of the two lines, cause this contradicts either $\rho \leq \lambda_{1}$, or the fact that $\rho\left(a_{2}, \ldots, a_{n-1}, s^{i}\right)$ is the first time that the line parallel to the $x_{1}$ direction hits $\widetilde{S}$, for either $i=1$ or $i=2$. We deduce $D$ is mapped into E.

Now assume $G=E \backslash F(D)$ is nonempty, and contains a point $p=\left(p_{1}, \ldots, p_{n}\right)$. If $Q_{p}$ contains a point $x \in O_{1} \backslash F(D)$, following the curve $t \rightarrow\left(t, x_{2}, \ldots, x_{n}\right)$ backwards from $x=\left(x_{1}, x_{2}, \ldots, x_{n}\right)$, we must hit either a point in the image of $\left.\eta^{i}\right|_{\left(-\varepsilon_{1}, a_{1}\right)}$ (which is a contradiction with the fact that both $\left(t, \ldots, x_{n}\right)$ for $t<x_{1}$ and $\left(t, a_{2}, \ldots, a_{n-1}, s^{i}\right)$ for $t<a_{1}$ are in $A(S)$; see definition 2.1), or the point $q$ (which contradicts (6.3) $)$. Thus for any point $p \in G$, we have $Q_{p} \subset V \backslash O_{1}$. 
Now take a point $p \in \partial G$, and pick up a sequence approaching it from within $G$ and contained in a line with speed vector $\frac{\partial}{\partial y_{1}}$. By the above, the set $Q$ for points in this sequence is contained in $V \backslash O_{1}$. We can take a subsequence carrying a convergent sequence of vectors, and thus $R_{p}$ has a vector of the form $d F_{x^{*}}(r)$ for $x^{*} \in D \subset O$. This violates the balanced condition, because of (6.2). This implies $\partial G=\emptyset$, thus $G=\emptyset$ because $E$ is connected and $F(D) \neq \emptyset$.

Finally, we claim there are no vectors coming from $V \backslash O_{1}$ in $R_{p}$ for $p \in \operatorname{int}(E)$. The argument is as above, but we now approach a point with a vector from $V \backslash O_{1}$ in $R_{p}$ within $E=F(D)$ and with speed $-\frac{\partial}{\partial y_{1}}$. The approaching sequence may be chosen so that it carries a convergent sequence of vectors from $F(D)$, and again (6.2) gives a contradiction with the balanced condition.

We now compute:

$$
\tilde{u}\left(x^{1}\right)-\tilde{u}\left(x^{2}\right)=\int_{s_{1}}^{s_{2}} \frac{d(\tilde{u} \circ \sigma)}{d s}=\int_{\sigma} d \tilde{u}
$$

The curve $F \circ \sigma$ runs through points of $S$. If $F(\sigma(s))$ is a cleave point, then $F \circ \sigma$ is a smooth curve near $s$. We show that cleave points are the only contributors to the above integral. If a point is not cleave, either it is the image of a conjugate vector, or has more than 2 incoming geodesics. As $F \circ \sigma$ maps into $\operatorname{int}(E)$, all vectors in $R_{F(\sigma(s))}$ come from $O$.

Let $N$ be the set of $s$ such that $\sigma(s)$ is conjugate. We notice that $\sigma(s)$ is not an A2 point for $s \in N$. This is proposition 6.2 in $\mathrm{AG}$, and is a standard result for cut loci in Riemannian manifolds. This means that at those points the kernel of $d F$ is contained in the tangent to $\widetilde{S}$. The intersection of $\widetilde{S}$ with the plane $H_{a}$ is the image of the curve $\sigma$. Thus, for $s \in N$ the tangent to the curve $\lambda_{1}$ is the kernel of $d_{\sigma(s)} F$. If $\sigma$ is differentiable at a point $s$ we deduce, thanks to 4.1, that the tangent to the curve $\lambda_{1}$ is the kernel of $d_{\sigma(s)} F$.

We now use a variation of length argument to get a variant of the Finsler Gauss lemma. Let $c=(l, w)$ be a tangent vector to $V \subset \mathbb{R} \times \partial \Omega$ at the point $x=(t, z)$, and assume $d_{x} F(c)=0$. We show that this implies $d \tilde{u}(c)=0$. Let $\gamma_{s}$ be a variation through geodesics with initial point in $z(s) \in \partial \Omega$ and the characteristic vector field at $z(s)$ as the initial speed vector, such that $\frac{\partial}{\partial s} z(s)=w$, and with total length $t+s l$. By the first variation formula and the equation for the characteristic vector field at $\partial \Omega$, the variation of the length of the curve $\gamma_{s}$ is $\frac{\partial \varphi}{\partial v}\left(p, d_{x} F\left(r_{x}\right)\right)$. $d_{x} F(c)-\frac{\partial \varphi}{\partial v}\left(p, d_{z} F(r)\right) \cdot w=-d g(w)$, and by the definition of $\gamma_{s}$, it is also $l$. We deduce $l=-d g(w)$, and thus $d \tilde{u}(c)=l+d g(w)=0$.

It follows that $d_{\sigma(s)} F\left(\sigma^{\prime}(s)\right)=0$ at points $s \in N$ where $\sigma$ is differentiable. As $\sigma$ is Lipschitz, the set of $s$ where it is not differentiable has measure 0 , and we deduce:

$$
\int_{N} d \tilde{u}\left(\sigma^{\prime}\right)=0
$$

$N$ is contained in the set of points where $d(F \circ \sigma)$ vanishes. Thus, by the Sard-Federer theorem, the image of $N$ has Hausdorff dimension 0 .

Let $\Sigma_{2}$ be the set of points in $L_{a}$ with more than 2 incoming geodesics. From the proof of [AG, 7.3], we see that the tangent to $\Sigma_{2}$ has dimension 0 and thus $\Sigma_{2}$ has Hausdorff dimension 0.

As $F$ is non-singular at points in $\left[s_{1}, s_{2}\right] \backslash N$, the set of $s$ in $\left[s_{1}, s_{2}\right] \backslash N$ mapping to a point in $\Sigma_{2} \cup N$ has measure zero.

Altogether, we see that the integral (6.4) can be restricted to the set $C$ of $s$ mapping to a cleave point. $C$ is an open set and thus can be expressed as the disjoint union of a countable amount of intervals. Let $A_{1}$ be one of those intervals. It is mapped by $F \circ \sigma$ diffeomorphically onto a smooth curve $c_{0}$ of cleave points contained in $L_{a}$. Points of the form $\left(t, a_{2}, \ldots, a_{n-1}, s\right)$ for $t<\tilde{t}\left(a_{2}, \ldots, a_{n-1}, s\right)$ map through $F$ to a half open ball in $E$. There must be points of $D$ mapping 
to the other side of $c_{0}$. Because of all the above, $c_{0}$ is also the image of other points in $\left[s_{1}, s_{2}\right]$. As $c$ is made of cleave points, it must be the image of another component of $C$, which we call $B_{1}$, also mapping diffeomorphically onto $c_{0}$. Choose a new component $A_{2}$, which is matched to another component $B_{2}$, different from the above, and so on, till the $A_{i}$ and $B_{i}$ are all the components of $C$.

We can write the integral on $B_{i}$ as an integral on $A_{i}$ (we add a minus sign, because the curve is traversed in opposite directions):

$$
\int_{A_{i}} d \tilde{u}\left(\sigma^{\prime}\right)+\int_{B_{i}} d \tilde{u}\left(\sigma^{\prime}\right)=\int_{A_{i}} d \tilde{u}_{l}\left(\sigma^{\prime}\right)-d \tilde{u}_{r}\left(\sigma^{\prime}\right)
$$

where $d \tilde{u}_{l}$ and $d \tilde{u}_{r}$ are the values of $d \tilde{u}$ computed from both sides. The balanced condition implies $\sigma^{\prime} \in \operatorname{ker}\left(d \tilde{u}_{l}-d \tilde{u}_{r}\right)$, and thus the above integral vanishes. The integral (6.4) is absolutely convergent by Lemma 2.14, and the proof follows.

Remark. The above proof took some inspiration from [H, 5.2]. The reader may be interested in James Hebda's tree-like curves.

\subsection{Structure of S near cleave and crossing points}

In this section we prove some more results about the structure of a balanced split locus near degenerate cleave and crossing points. Besides their importance for proving that $\partial T=0$, we believe they are interesting in their own sake.

Lemma 6.3. Let $p \in S$ be a (possibly degenerate) cleave point, and let $Q_{p}=\left\{x^{1}, x^{2}\right\}$.

There are disjoint univocal neighborhoods $O_{1}$ and $O_{2}$ of $x^{1}$ and $x^{2}$, and a neighborhood $U$ of $p$ such that for any $q \in U, Q_{q}$ is contained in $\mathrm{O}_{1} \cup \mathrm{O}_{2}$.

Furthermore, if we define:

$$
A_{i}=\left\{q \in U \text { such that } Q_{q} \cap O_{i} \neq \emptyset\right\}
$$

for $i=1,2$, then $A_{1} \cap A_{2}$ is the graph of a Lipschitz function, for adequate coordinates in $U$.

Proof. The points $x^{1}$ and $x^{2}$ are at most of first order, so we can take univocal neighborhoods $O_{1}$ and $O_{2}$ of $x^{1}$ and $x^{2}$. By definition of $Q_{p}$ and the compactness of $\Omega$, we can achieve the first property, reducing $U$ if necessary.

We know $\widehat{d_{x^{1}} F(r)}$ is different from $\widehat{d_{x^{2}} F(r)}$. For fixed arbitrary coordinates in $U$, we can assume that $\left\{\widehat{d_{x} F(r)}\right.$ for $\left.x \in O_{1}\right\}$ can be separated by a hyperplane from $\left\{\widehat{d_{x} F(r)}\right.$ for $\left.x \in O_{2}\right\}$, after reducing $U, O_{1}, O_{2}$ if necessary. Therefore, there is a vector $Z_{0} \in T_{p} \Omega$ and a number $\delta>0$ such that

$$
\widehat{d_{x} F(r)}(Z)<\widehat{d_{x^{\prime}} F(r)}(Z)+\delta \quad \forall x \in O_{1}, x^{\prime} \in O_{2}
$$

for any unit vector $Z$ in a neighborhood $G$ of $Z_{0}$. Let $C^{+}=\{t Z: t>0, Z \in G\}$ be a one-sided cone containing $Z$. We write $q+C^{+}$for the cone displaced to have a vertex in $q$.

Choose $q \in A_{1} \cap A_{2}$, and $Z \in G$. Let $\mathcal{R}=\left\{q^{\prime} \in U: q^{\prime}=q+t Z, t>0\right\}$ be a ray contained in $\left(q+C^{+}\right) \cap U$. We claim $\mathcal{R} \subset A_{1} \backslash A_{2}$.

For two points $q_{1}=q+t_{1} Z, q_{2}=q+t_{2} Z \in \mathcal{R}$, we say $q_{1}<q_{2}$ if and only $t_{1}<t_{2}$. If $\mathcal{R} \cap A_{2} \neq \emptyset$, let $q_{0}$ be the infimum of all points $p>0$ in $\mathcal{R} \cap A_{2}$, for the above order in $\mathcal{R}$. If $q_{0} \in A_{1}$ (whether $q_{0}=q$ or not), we can approach $q_{0}$ with a sequence of points $q_{n}=F\left(x_{n}\right)>q_{0}$ carrying vectors 
$d_{x_{n}} F(r)$ with $x_{n} \in O_{2}$. The limit point of this sequence is $q_{0}$, and the limit vector is $d_{x} F(r)$ for some $x \in \mathrm{O}_{2}$, but the incoming vector is in $-G$, which contradicts the balanced condition by (6.5).

If $q_{0} \in A_{2} \backslash A_{1}$, then approaching $q_{0}$ with points $q<q_{n}=F\left(x_{n}\right)<q_{0}$, we get a new contradiction with the balanced property. The only possibility is $\mathcal{R} \subset A_{1} \backslash A_{2}$. As the vector $Z$ is arbitrary, we have indeed $\left(q+C^{+}\right) \cap U \subset A_{1} \backslash A_{2}$.

Fix coordinates in $U$, and let $\varepsilon=\frac{1}{2} \operatorname{dist}(p, \partial U)$. Let $B_{\varepsilon}$ be the ball of radius $\varepsilon$ centered at $p$. By the above, the hypothesis of lemma 3.7 are satisfied, for $A=A_{1} \backslash A_{2}$, the cone $C^{+}$, the number $\varepsilon$, and $V=B_{\varepsilon}$. Thus, we learn from lemma 3.7 that $A_{1} \cap A_{2} \cap B_{\varepsilon}$ is the graph of a Lipschitz function along the direction $Z_{0}$ from any hyperplane transversal to $Z_{0}$.

The following three lemmas contain more detailed information about the structure of a balanced split locus near a crossing point. The following is stated for the case $n>2$, but it holds too if $n=2$, though then $L$ reduces to a single point $\{a\}$.

Definition 6.4. The normal to a subset $X \subset T_{p}^{*} \Omega$ is the set of vectors $Z$ in $T_{p} \Omega$ such that $\omega(Z)$ is the same number for all $\omega \in X$.

Lemma 6.5. Let $p \in S$ be a crossing point. Let $B \subset T_{p}^{*} \Omega$ be the affine plane spanned by $R_{p}^{*}$. Let $L$ be the normal to $B$, which by hypothesis is a linear space of dimension $n-2$, and let $C$ be a (double-sided) cone of small amplitude around $L$.

There are disjoint univocal open sets $O_{1}, \ldots, O_{N} \subset V$ and an open neighborhood $U$ of $p$ such that $Q_{q} \subset \cup_{i} O_{i}$ for all $q$ in $U$.

Furthermore, define sets $A_{i}$ as in lemma 6.3, and call $\mathcal{S}=\cup_{i, j} A_{i} \cap A_{j}$ the essential part of $S$. Define $\Sigma=\cup_{i, j, k} A_{i} \cap A_{j} \cap A_{k}$ and let $\mathcal{C}=\mathcal{S} \backslash \Sigma$.

(1) At every $q \in \Sigma$, there is $\varepsilon>0$ such that $\Sigma \cap\left(q+B_{\varepsilon}\right) \subset q+C$.

(2) $\Sigma$ itself is contained in $p+C$.

The next lemma describes the intersection of $\mathcal{S}$ with 2-planes transversal to $L$.

Lemma 6.6. Let $p \in S$ be a crossing point as above. Let $P \subset T_{p} \Omega$ be a 2-plane intersecting $C$ only at the origin, and let $P_{a}=P+a$ be a 2-plane parallel to $P$ for $a \in L$.

1. If $|a|<\varepsilon_{1}$, the intersection of $\mathcal{S}$, the plane $P_{a}$, and $U$ is a connected Lipschitz tree.

2. The intersection of $\mathcal{S}$, the plane $P_{a}$, and the annulus of inner radius $c \cdot|a|$ and outer radius $\varepsilon_{2}$ :

$$
A\left(c|a|, \varepsilon_{2}\right)=\left\{q \in U: c|a|<|q|<\varepsilon_{2}\right\}
$$

is the union of $N$ Lipschitz arcs separating the sets $A_{i}$.

Remark. We cannot say much about what happens inside $P_{a} \cap B(P, c|a|)$. The segments in $P_{a} \cap A\left(c|a|, \varepsilon_{2}\right)$ must meet together, yielding a connected tree, but this can happen in several different ways (see figure 2).

Finally, we can describe the connected components of $\mathcal{C}=\mathcal{S} \backslash \Sigma$ within $U$ :

Lemma 6.7. Under the same hypothesis, for every $i=1, \ldots, N$ there is a coordinate system in $U$ such that: 

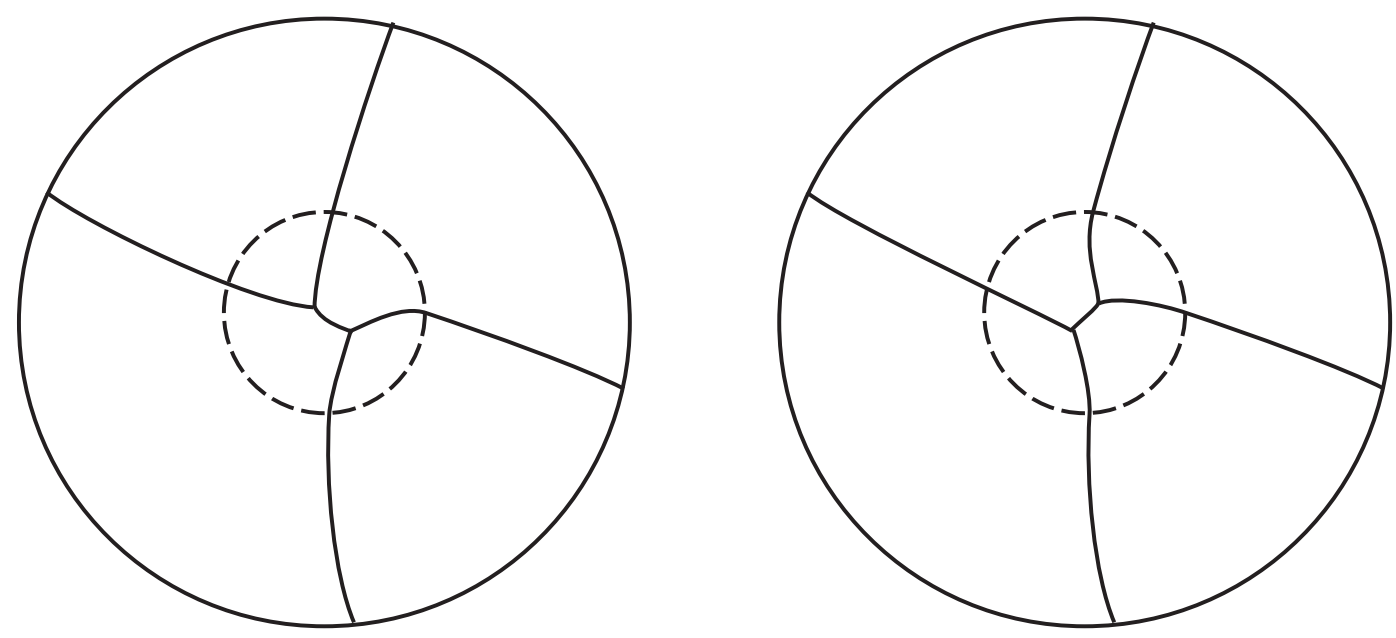

Figure 2: Two possible intersections of a plane $P_{a}$ with $\mathcal{S}$

- The set $\partial A_{i}$ is the graph of a Lipschitz function $h_{i}$, its domain delimited by two Lipschitz functions $f_{l}$ and $f_{r}$, for $L^{*} \subset L$ :

$$
\partial A_{i}=\left\{\left(a, t, h_{i}(t)\right), a \in L^{*}, f_{l}(a)<t<f_{r}(a)\right\}
$$

- A connected component $\mathcal{C}_{0}$ of $\mathcal{C}$ contained in $\partial A_{i}$ admits the following expression, for Lipschitz functions $f_{1}$ and $f_{2}$, for $L_{0} \subset L$ :

$$
\mathcal{C}_{0}=\left\{\left(a, t, h_{i}(t)\right), a \in L_{0}, f_{1}(a)<t<f_{2}(a)\right\}
$$

Corollary 6.8. $\mathcal{H}^{n-2}(\Sigma)<\infty$.

Proof of corollary. We apply the general area-coarea formula (see [F, 3.2.22]), with $W=\Sigma, Z=L$, and $f$ the projection from $U$ onto $L$ parallel to $P$, and $m=\mu=\nu=n-2$, to learn:

$$
\int_{\Sigma} a p J f d \mathcal{H}^{n-2}=\int_{L} \mathcal{H}^{0}\left(f^{-1}(\{z\})\right) d \mathcal{H}^{n-2}(z)=\int_{L} \mathcal{H}^{0}\left(\Sigma \cap P_{a}\right) d \mathcal{H}^{n-2}(a)
$$

ap $\left.J f\right|_{\Sigma}$ is bounded from below, so if we can bound $\mathcal{H}^{0}\left(\Sigma \cap P_{a}\right)$ uniformly, we get a bound for $\mathcal{H}^{n-2}(\Sigma)$.

The set $\mathcal{C} \cap P_{a} \cap U$ is a simplicial complex of dimension 1, and a standard result in homology theory states that the number of edges minus the number of vertices is the same as the difference between the homology numbers of the complex: $h^{1}-h^{0}$. The graph is connected and simply connected, so this last number is -1 . The vertices of $\mathcal{C} \cap P_{a} \cap U$ consist of $N$ vertices of degree 1 lying at $\partial U$ and the interior vertices having degree at least 3 . The handshaking lemma states that the sum of the degrees of the vertices of a graph is twice the number of edges, so we get the inequality $2 e \geq N+3 \bar{v}$ for the number $e$ of edges and the number $\bar{v}$ of interior vertices. Adding this to the previous equality $e-(N+\bar{v})=-1$, we get $\bar{v} \leq N-2$. We have thus bounded $\bar{v}=\mathcal{H}^{0}\left(\Sigma \cap P_{a}\right)$ with a bound valid for all $a$. 
Proof of 6.5. This lemma can be proven in a way similar to 6.3, but we will take some extra steps to help us with the proof of the other lemmas.

First, recall the map $\Delta$ defined in (3.4). Each point $x$ in $\Delta^{-1}\left(R_{p}^{*}\right)$ has a univocal neighborhood $\mathcal{O}_{x}$. Recall $R_{p}^{*}$ consists only of covectors of norm 1 . Let $\gamma$ be the curve obtained as intersection of $B$ and the covectors of norm 1. Instead of taking the neighborhoods $\mathcal{O}_{x}$ right away, which would be sufficient for this lemma, we cover $R_{p}^{*}$ with open sets of the form $\Delta\left(\mathcal{O}_{x}\right) \cap \gamma$.

By standard results in topology, we can extract a finite refinement of the covering of $R_{p}^{*} \subset \gamma$ by the sets $\Delta\left(\mathcal{O}_{x}\right) \cap \gamma$ consisting of disjoint non-empty intervals $I_{1}, \ldots, I_{N}$. Let $\tilde{I}_{i}$ be the set of points $t x$ for $t \in\left(1-\varepsilon_{1}, 1+\varepsilon_{1}\right)$ and $x \in I_{i}$, and choose a linear space $M_{0}$ of dimension $n-2$ transversal to $B$. Define the sets of our covering:

$$
O_{i}=\Delta^{-1}\left(\tilde{I}_{i}+B\left(M_{0}, \varepsilon_{2}\right)\right)
$$

for the ball of radius $\varepsilon_{2}$ in $M_{0}\left(\varepsilon_{1}\right.$ and $\varepsilon_{2}$ are arbitrary, and small).

We can assume that $Q_{q} \subset \cup_{i} O_{i}$ for all $q$ in $U$ by reducing $U$ and the $O_{i}$ further if necessary, hence we only need to prove the two extra properties to conclude the theorem.

The approximate tangent to $\Sigma$ at a point $q \in \Sigma \cap U$ is contained in the normal to $R_{q}^{*}$ (see the definition of approximate tangent in $\mathrm{AG}$ and use the proof of proposition 7.3 there, or merely use the balanced property). If $R_{q}^{*}$ is contained in a sufficiently small neighborhood of $\gamma$ and contains points from at least three different $I_{i}$, its normal must be close to $L$. Thus if we chose $\varepsilon_{1}$ and $\varepsilon_{2}$ small enough, the approximate tangent to $\Sigma \cap U$ at a point $q \in \Sigma$ is contained in $C$. If property (1) did not hold for any $\varepsilon$ at a point $q$, we could find a sequence of points converging to $q$ whose directions from $q$ would remain outside $C$, violating the above property.

Finally, the second property holds if we replace $U$ by $U \cap B_{\varepsilon}$, for the number $\varepsilon$ that appears when we apply property (1) to $p$.

Proof of 6.6. Just like in 6.3, we can assume that each set $\left\{\widehat{d_{x} F(r)}\right.$ for $\left.x \in O_{i}\right\}$ can be separated from the others by a hyperplane (e.g., a direction $Z_{i}$ ), such that:

$$
\widehat{d_{x} F(r)}(Z)<\widehat{d_{x^{\prime}} F(r)}(Z)+\delta \quad \forall x \in O_{i}, x^{\prime} \in O_{j}, i \neq j
$$

for some $\delta>0$ and any unit vector $Z$ in a neighborhood $G_{i}$ of $Z_{i}$. Thanks to the care we took in the proof of the previous lemma, we can assume all $Z_{i}$ belong to the plane $P$ in the statement of this lemma: indeed the intervals $I_{i}$ can be separated by vectors in any plane transversal to $L$, and the sets $\Delta\left(O_{i}\right)$ are contained in neighborhoods of the $I_{i}$.

Define the one-sided cones $C_{i}^{+}=\left\{t Z: t>0, Z \in G_{i}\right\}$. The above implies that the intersection of each $C_{i}^{+}$with $P$ is a nontrivial cone in $P$ that consists of rays from the vertex.

By the same arguments in 6.3, we can be sure that whenever $q \in A_{i}$, then $\left(q+C_{i}^{+}\right) \cap U \subset A_{i}$. This implies that $\partial A_{i}$ is the graph of a Lipschitz function along the direction $Z_{i}$ from any hyperplane transversal to $Z_{i}$. We notice $\partial A_{i}$ is (Lipschitz) transversal to $P$, so for any $a \in L, \partial A_{i} \cap P$ is a Lipschitz curve. As the cone $C$ is transversal to $P$, and the tangent to $\Sigma$ is contained in $C$, we see $\Sigma \cap P_{a}$ consists of isolated points.

Thus $\mathcal{S} \cap P_{a}$ is a Lipschitz graph and $\Sigma \cap P_{a}$ is the set of its vertices. If it were not a tree, there would be a bounded open subset of $P_{a} \cap U \backslash \mathcal{S}$ with boundary contained in $\mathcal{S}$. An interior point $q$ belongs to some $A_{k}$. Then the cone $q+C_{k}^{+}$is contained in $A_{k}$, but on the other hand its intersection with $P_{a}$ contains a ray that must necessarily intersect $\mathcal{S}$, which is a contradiction.

We notice $P_{a} \cap\left(p+C_{i}^{+}\right) \subset A_{i}$. This set is a cone in $P_{a}$ (e.g. a circular sector) with vertex at most a distance $c_{1}|a|$ from $p+a$, where $c_{1}>0$ depends on the amplitude of the different $C_{i}$. 


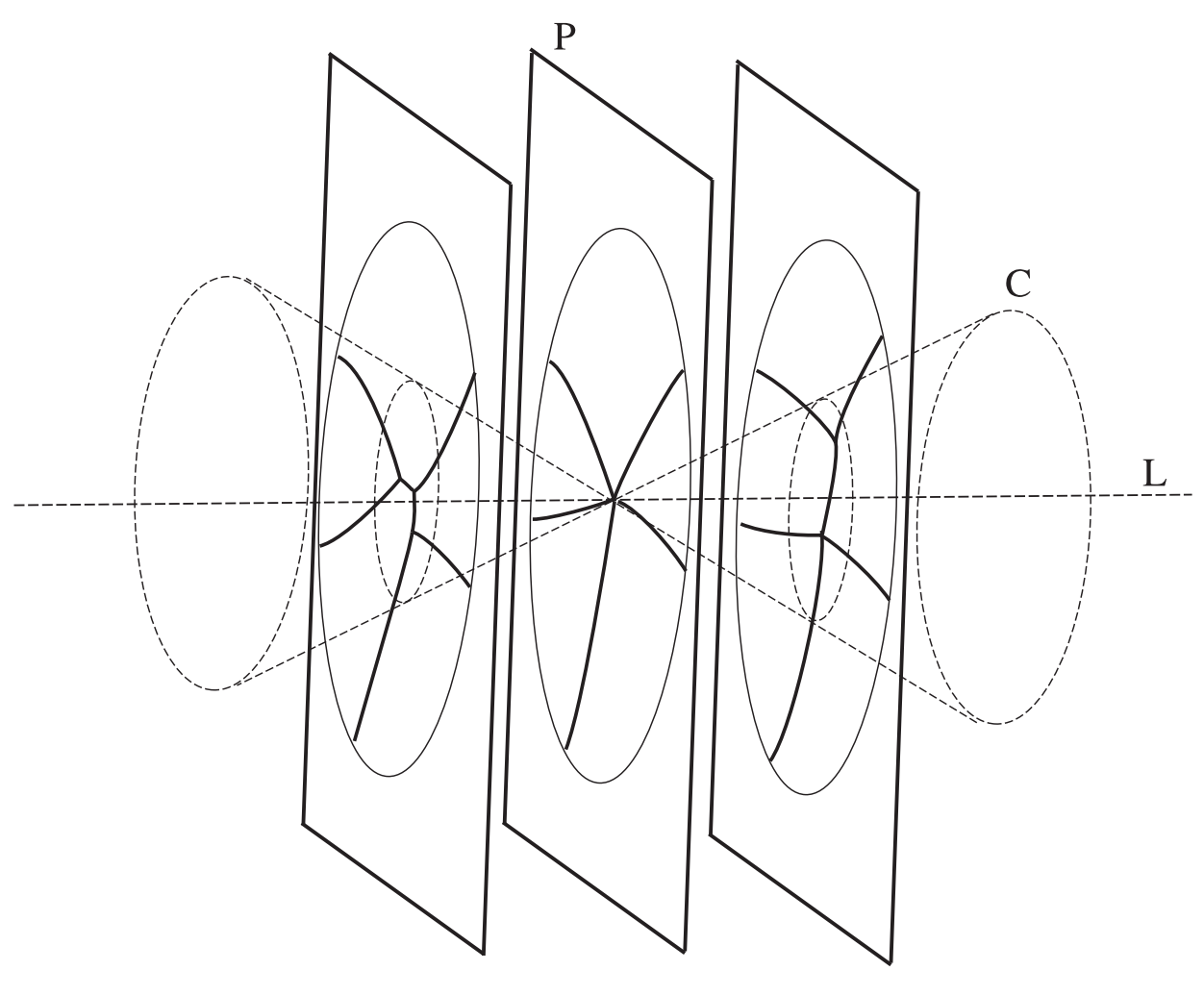

Figure 3: $\mathcal{S}$ near a crossing point

If $a=0$, the $N$ segments departing from $p$ with speeds $Z_{i}$ belong to each $A_{i}$ respectively. Let us assume that the intervals $I_{i}$ appearing in the last proof are met in the usual order $I_{1}, I_{2} \ldots, I_{N}$ when we run along $\gamma$ following a particular orientation, and call $P^{i}$ the region delimited by the rays from $p$ with speeds $Z_{i}$ and $Z_{i+1}\left(\right.$ read $Z_{1}$ instead of $Z_{N+1}$ ).If there is a point $q \in P^{i} \cap A_{k} \cap B\left(\varepsilon_{2}\right)$ for sufficiently small $\varepsilon_{2}$, then $\left(q+C_{k}^{+}\right) \cap U$ would intersect either $p+C_{i}^{+}$or $p+C_{i+1}^{+}$, and yield a contradiction if $k$ is not $i$ or $i+1$. Thus $P^{i} \subset A_{i} \cup A_{i+1}$. Clearly there must be some point $q$ in $P_{i} \cap A_{i} \cap A_{i+1}$, to which we can apply lemma 6.3. $A_{i} \cap A_{i+1}$ is a Lipschitz curve near $q$ transversal to $Z_{i}$ (and to $Z_{i+1}$ ), and it cannot turn back. The curve does not meet $\Sigma$, and it cannot intersect the rays from $p$ with speeds $Z_{i}$ and $Z_{i+1}$, so it must continue up to $p$ itself. For any $q \in A_{i} \cap A_{i+1}$, the cone $q+C_{i}^{+}$is contained in $A_{i}$, and the cone $q+C_{i+1}^{+}$is contained in $A_{i+1}$. This implies there cannot be any other branch of $A_{i} \cap A_{i+1}$ inside $P_{i}$.

This is all we need to describe $\mathcal{S} \cap P \cap B\left(\varepsilon_{2}\right)$ : it consists of $N$ Lipschitz segments starting at $p$ and finishing in $P \cap \partial B\left(\varepsilon_{2}\right)$. The only multiple point is $p$.

For small positive $|a|$, we know by condition (2) of the previous lemma that $P_{a} \cap \Sigma \subset C \cap P_{a}=$ $B\left(c_{2}|a|\right) \cap P_{a}$ for some $c_{2}>0$. Similarly as above, define regions $P_{a}^{i} \subset P_{a} \cap A\left(c|a|, \varepsilon_{2}\right)$ delimited by the rays from $a$ with directions $Z_{i}$ and $Z_{i+1}$, and the boundary of the ring $A\left(c|a|, \varepsilon_{2}\right)$, for a constant $c>\max \left(c_{1}, c_{2}\right)$. Take $c$ big enough so that for any $q \in P_{a}^{i}$ and any $k \neq i, i+1$, $q+C_{k}^{+} \cap U \cap P_{a}$ intersects either $p+C_{i}^{+}$or $p+C_{i+1}^{+}$. The same argument as above shows that $A_{i} \cap A_{i+1} \subset P_{a}^{i} \subset A_{i} \cup A_{i+1}$. We conclude there must be a Lipschitz curve of points of $A_{i} \cap A_{i+1}$, which starts in the inner boundary of $A\left(c|a|, \varepsilon_{2}\right)$, and ends up in the outer boundary.

Proof of 6.7. First we assume $U$ has a product form $U=L^{*} \times P^{*}$ for open $\operatorname{discs} L^{*} \subset L$ and 


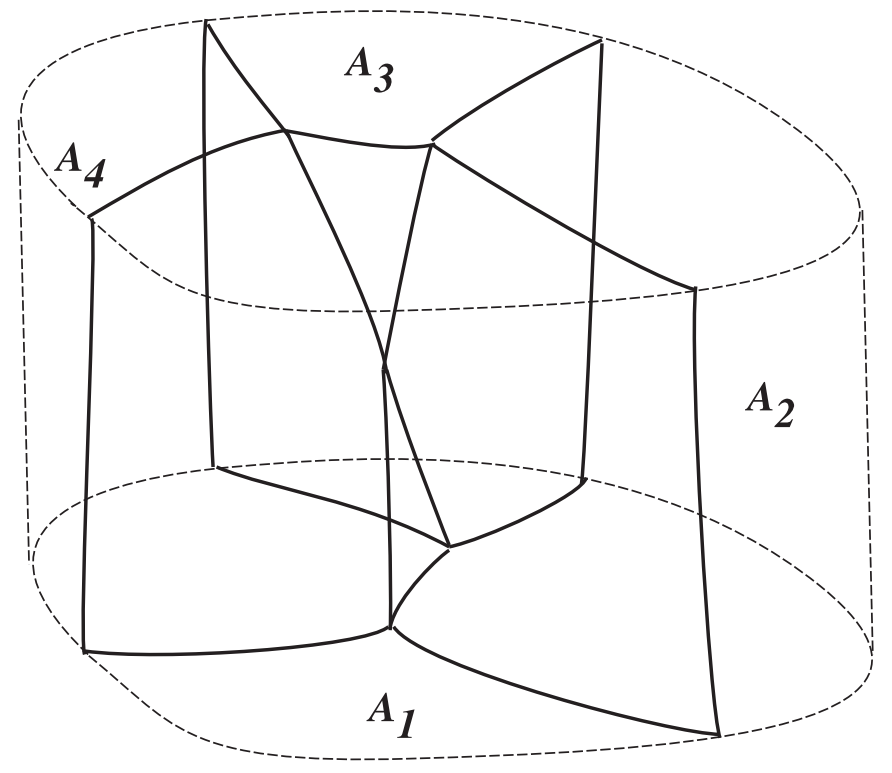

Figure 4: A neighborhood of a crossing point (this view is rotated with respect to figure 3)

$P^{*}=B\left(P, \varepsilon_{2}\right) \subset P$.

Recall $\partial A_{i}$ is the graph of a Lipschitz function along the direction $Z_{i}$ from any hyperplane transversal to $Z_{i}$. Let $H_{i}=L+W$ be one such hyperplane that contains the subspace $L$ and the vector line $W \subset P$, and construct coordinates $L \times W \times<Z_{i}>$. It follows from the previous lemma that $\partial A_{i} \cap P_{a}^{*}$ is a connected Lipschitz curve. In these coordinates $\partial A_{i}$ is the graph of a Lipschitz function $h_{i}$. Its domain, for fixed $a$, is a connected interval, delimited by two functions $f_{l}: L^{*} \rightarrow W$ and $f_{r}: L^{*} \rightarrow W$. Condition (1) of lemma 6.5 assures they are Lipschitz.

A connected component $\mathcal{C}_{0}$ of $\mathcal{C}$ is contained in only one $A_{i} \cap A_{j}$. We can express it in the coordinates defined above for $\partial A_{i}$. The intersection of $\mathcal{C}_{0}$ with each plane $P_{a}$ is either empty or a connected Lipschitz curve. The second part follows as before.

\subsection{Conclusion}

Using lemma 6.2, we show without much effort that $\partial T$ vanishes near edge points. Using the structure results from the previous section, we show also that it vanishes at cleave points (including degenerate ones) and crossing points.

Proposition 6.9. Let $p \in S$ be an edge point. Then the boundary of $T$ vanishes near $p$.

Proof. Let $p$ be an edge point with $Q_{p}=\{x\}$. Let $O$ be a univocal neighborhood of $x$. It follows by a contradiction argument that there is an open neighborhood $U$ of $p$ such that $Q_{q} \subset O$ for all $q \in U$. Recall the definition of $T$ :

$$
T(\phi)=\sum_{j} \int_{\mathcal{C}_{j, 1}}\left(h_{1}-h_{2}\right) \phi
$$


For any cleave point $q \in U$ with $Q_{q}=\left\{x_{1}, x_{2}\right\}, h_{i}(q)=\tilde{u}\left(x_{i}\right)$. By the above, both $x_{1}$ and $x_{2}$ are in $O$. As $O$ is univocal, we see $h_{1}=h_{2}$ at $q$. The integrand of $T$ vanishes near $p$, and thus $\partial T=0$.

Proposition 6.10. Let $p \in S$ be a (possibly degenerate) cleave point. Then $\partial T$ vanishes near $p$.

Proof. Use the sets $U, A_{1}$ and $A_{2}$ of lemma 6.3 .

Whenever $\phi$ is a $n-1$ differential form with support contained in $U$, we can compute:

$$
T(\phi)=\int_{A_{1} \cap A_{2}}\left(h_{1}-h_{2}\right) \phi
$$

The components of cleave points inside either $A_{1}$ or $A_{2}$ do not contribute to the integral, for the same reasons as in the previous lemma. Recall the definition of $\partial T$, for a differential $n-2$ form $\sigma$ :

$$
\partial T(\sigma)=T(d \sigma)=\int_{A_{1} \cap A_{2}}\left(h_{1}-h_{2}\right) d \sigma
$$

We can apply a version of Stokes theorem that allows for Lipschitz functions. We will provide references for this later:

$$
T(d \sigma)=\int_{A_{1} \cap A_{2}} d\left(h_{1}-h_{2}\right) \sigma
$$

The balanced condition imposes that for any vector $v$ tangent to $A_{1} \cap A_{2}$ at a non-degenerate cleave point $q$ with $Q_{q}=\left\{x_{1}, x_{2}\right\}$.

$$
\hat{X}^{1}(v)=\hat{X}^{2}(v)
$$

for the incoming vectors $X^{i}=d_{x_{i}} F(r)$. Recall that $\mathcal{H}^{n-1}$-almost all points are cleave, and $d h_{i}$ is dual to the incoming vector $X^{i}$, so $T(d \sigma)=0$.

Proposition 6.11. Let $p \in S$ be a crossing point. Then the boundary of the current $T$ (defined in 5.2) vanishes near $p$.

Proof. We use lemma 6.7 to describe the structure of connected components of $\mathcal{C}$ near $p$. Let $\Sigma_{T}$, the set of higher order points, be the set of those points such that $R_{q}^{*}$ spans an affine subspace of $T_{q}^{*} \Omega$ of dimension greater than 2 .

Take any connected component $\mathcal{C}_{0}$ of $\mathcal{C}$ contained in $\partial A_{i} . \partial \mathcal{C}_{0}$ decomposes into several parts:

- The regular boundary, consisting of two parts $D_{1}$ and $D_{2}$ :

$$
\begin{aligned}
& D_{1}=\left\{\left(a_{1}, \ldots, a_{n-2}, f_{1}(a), h_{i}\left(f_{1}(a)\right)\right), \forall a \in L^{*} \text { such that } f_{l}(a)<f_{1}(a)<f_{2}(a)\right\} \\
& D_{2}=\left\{\left(a_{1}, \ldots, a_{n-2}, f_{2}(a), h_{i}\left(f_{2}(a)\right)\right), \forall a \in L^{*} \text { such that } f_{1}(a)<f_{2}(a)<f_{r}(a)\right\}
\end{aligned}
$$

- The points of higher order, or $\partial \mathcal{C}_{0} \cap \Sigma_{T}$.

- The singular boundary, or those points $q=\left(a_{1}, \ldots, a_{n-2}, f_{1}(a), h_{i}\left(f_{1}(a)\right)\right)$ where $f_{1}(a)=$ $f_{2}(a)$ and $R_{q}$ is contained in an affine plane.

- A subset of $\partial U$. 
Using a version of Stokes theorem that allows for Lipschitz functions, we see that

$$
\int_{\mathcal{C}_{0}} v d \sigma=\int_{\mathcal{C}_{0}} d(v \sigma)-\int_{\mathcal{C}_{0}}(d v) \sigma=\int_{D_{1}} v \sigma-\int_{D_{2}} v \sigma-\int_{\mathcal{C}_{0}}(d v) \sigma
$$

for any function $v$ and $n-2$ form $\sigma$ with compact support inside $U$. Indeed, the last coordinate of the parametrization of $\mathcal{C}_{0}$ is given by a Lipschitz function, so we can rewrite the integral as one over a subset of $L \times W$, and only Gauss-Green theorem is needed. We can apply the version in $\mathrm{F}$, 4.5.5], whose only hypothesis is that the current $\mathcal{H}^{n-1}\left\lfloor\partial \mathcal{C}_{0}\right.$ must be representable by integration. Using [F, 4.5.15] we find that it is indeed, because its support is contained in a rectifiable set Here we are assuming that $D_{1}$ is oriented as the boundary of $\mathcal{C}_{0}$, while $D_{2}$ is oriented in the opposite way, to match the orientation of $D_{1}$.

Notice we have discarded several parts of $\partial \mathcal{C}_{0}$ :

- A subset of $\partial \mathcal{C}_{0}$ inside $\partial U$ does not contribute to the integral because $\operatorname{supp}(\sigma) \subset \subset U$.

- $\partial \mathcal{C}_{0} \cap \Sigma_{T}$ does no contribute because it has Hausdorff dimension at most $n-3$.

- The singular boundary does not contribute either, because the normal to $\widetilde{\mathcal{C}_{0}}$ at a point of the singular boundary does not exist (see [F, 4.5.5]).

We now prove that $\partial T=0$.

For a form $\sigma$ of dimension $n-2$ and compact support inside $U$ :

$$
T(d \sigma)=\sum_{i} \int_{\mathcal{C}_{i}}\left(h_{l}-h_{r}\right) d \sigma=\sum_{i} \int_{\mathcal{C}_{i}} d\left(h_{l}-h_{r}\right) \sigma+\sum_{i}\left(\int_{D_{i, 1}}\left(h_{l}-h_{r}\right) \sigma-\int_{D_{i, 2}}\left(h_{l}-h_{r}\right) \sigma\right)
$$

where $D_{i, 1}$ and $D_{i, 2}$ are the two parts of the regular boundary of $\mathcal{C}_{i}$.

The first summand is zero and the remaining terms can be reordered (the sum is absolutely convergent because $h$ is bounded and $\mathcal{H}^{n-2}(\Sigma)$ is finite):

$$
\sum_{i}\left(\int_{D_{i, 1}}\left(h_{l}-h_{r}\right) \sigma-\int_{D_{i, 2}}\left(h_{l}-h_{r}\right) \sigma\right)=\int_{\Sigma \backslash \Sigma_{T}} \sum_{(i, j) \in I(q)}\left(h_{i, j, l}-h_{i, j, r}\right) \sigma d q
$$

where every point $q \in \Sigma \backslash \Sigma_{T}$ has a set $I(q)$ consisting of those $i$ and $j=1,2$ such that $q$ is in the boundary part $D_{j}$ of the component $\mathcal{C}_{i}$. The integrand at point $q$ is then:

$$
\sigma \sum_{(i, j) \in I(q)}\left(h_{i, j, l}-h_{i, j, r}\right)
$$

where $h_{i, j, l}$ is the value of $\tilde{u}(x)$ coming from the side $l$ of component $\mathcal{C}_{i}$ and boundary part $D_{j}$.

By the structure lemma 3.5, we can restrict the integral to crossing points. Let $O_{1}, \ldots, O_{N}$ be the disjoint univocal sets that appear when we apply 6.5 to $p$. For a crossing point $q, I(q)$ is in correspondence with the set of indices $k$ such that $O_{k} \cap Q_{p} \neq \emptyset$. Indeed, the intersection of $\mathcal{S}$ with the plane $P_{a}$ containing $q$ is a Lipschitz tree, and $q$ is a vertex, and belongs to the regular boundary of the components that intersect $P_{a}$ in an edge. The $h_{i, j, l}$ in the sum appear in pairs: one is the value from the left coming from one component $\mathcal{C}_{i}$ and the value from the right of another component $\mathcal{C}_{i^{\prime}}$. Each one comes from a different side, so they carry opposite signs, and they cancel. The integrand at $q$ vanishes altogether, so $\partial T=0$. 


\section{References}

[AG] P. Angulo, L. Guijarro, Cut and singular loci up to codimension 3, http://arxiv.org/abs/0806.2229

[BCS] D. Bao, S. S. Chern, Z. Shen, An introduction to Riemann-Finsler geometry, Graduate Texts in Mathematics, 200, Springer.

[CS] P. Cannarsa, C. Sinestrari, Semiconcave Functions, Hamilton-Jacobi Equations, and Optimal Control, Progress in Nonlinear Differential Equations and Their Applications, 58, Birkhäuser

[CR] M. Castelpietra, L. Rifford, Regularity properties of the distance functions to conjugate and cut loci for viscosity solutions of Hamilton-Jacobi equations and applications in Riemannian geometry arXiv:0812.4107v1 (2008)

[D] J. J. Duistermaat, Fourier Integral Operators, Birkhäuser, Boston, Progress in Mathematics, 130 (1996)

[F] H. Federer, Geometric measure theory, Springer-Verlag New York Inc., New York, 153 (1969).

[GG] M. Golubitsky, V. Guillemin Stable Mappings and their Singularities Graduate Texts in Mathematics, 14 Springer-Verlag

[Ha] A. Hatcher. Algebraic topology. Cambridge University Press.

http://www.math.cornell.edu/ hatcher

[H] J. Hebda, Parallel translation of curvature along geodesics, Trans. Amer. Math. Soc., Vol. 299, No 2 (Feb., 1987), 559-572.

[IT] J. Itoh, M. Tanaka. The Lipschitz continuity of the distance function to the cut locus. Transactions of the A.M.S. 353 (2000), no. 1, 21-40

[LN] YY.Li, L. Nirenberg, The distance function to the boundary, Finsler geometry, and the singular set of viscosity solutions of some Hamilton-Jacobi equations. Comm. Pure Appl. Math. 58 (2005), no. 1, 85-146.

[L] P. L. Lions, Generalized solutions of Hamilton-Jacobi equations Research Notes in Mathematics 69, Pitman, London (1982).

[MM] C. Mantegazza, A. C. Mennucci, Hamilton-Jacobi Equations and Distance Functions on Riemannian Manifolds Appl. Math. Optim. 47 (2003), pp.1-25

[Me] A. C. Mennucci, Regularity And Variationality Of Solutions To Hamilton-Jacobi Equations. Part I: Regularity (2nd Edition), ESAIM Control Optim. Calc. Var. 13 (2007), no. 2, 413-417

[W] F.W.Warner, The conjugate locus of a Riemannian manifold, Amer.J. of Math. 87 (1965) 575-604. MR 34:8344 